\title{
Dynamics in coalescing critical layers
}

\author{
By N. J. BALMFORTH ${ }^{1}$, C. PICCOLO ${ }^{1}$ \\ AND O. M. UMURHA $\mathbf{N}^{2}$ \\ ${ }^{1}$ Department of Applied Mathematics and Statistics, School of Engineering, \\ University of California at Santa Cruz, CA 95064, USA \\ ${ }^{2}$ Center for Turbulence Research, Stanford University, CA 94305-3030, USA
}

(Received 3 January 2001 and in revised form 21 May 2001)

This article continues an exploration of instabilities of jets in two-dimensional, inviscid fluid on the beta-plane. At onset, for particular choices of the physical parameters, the normal modes responsible for instability have critical levels that coalesce along the axis of the jet. Matched asymptotic expansion (critical layer theory) is used to derive a reduced model describing the dynamics of these modes. Because the velocity profile is locally parabolic in the vicinity of the critical levels the dynamics is richer than in standard critical layer problems. The model captures the inviscid saturation of unstable modes, the excitation of neutral Rossby waves, and the decay of disturbances when there are no discrete normal modes. Inviscid saturation occurs when the vorticity distribution twists up into vortical structures that take the form of either a pair of 'cat's eye' patterns straddling the jet axis, or a single row of vortices. The addition of weak viscosity destroys these cat's eye structures and causes the critical layer to spread diffusively. The results are compared with numerical simulations of the governing equations.

\section{Introduction}

In paper I (Balmforth \& Piccolo 2001), we considered the onset of meandering in an unstable, nearly inviscid jet. Such flows are commonly used as models of wakes behind obstacles, geophysical currents and laboratory experiments in rotating annuli (e.g. Solomon, Holloway \& Swinney 1993), and display a rich variety of complex fluid dynamical behaviour. A specific issue of recent interest has been the transport and mixing in the resulting unsteady flows (e.g. del Castillo-Negrete \& Morrison 1993), a problem of notable geophysical importance. But a previously unexplored question was how weak linear instabilities saturate into finite-amplitude meanders, a question we addressed in paper I.

In paper I, we focused on a Bickley jet (a two-dimensional flow with a $\operatorname{sech}^{2} y$ velocity profile, where $y$ is the cross-stream coordinate) of incompressible fluid on the beta-plane. The main difficulty in the analysis is that superficial singularities arise at the critical levels of neutral waves (the levels along which the wave speed matches the flow). These singularities reflect how the solution develops unresolved sharp spatial gradients in narrow regions surrounding the critical levels (the critical layers). Matched asymptotics are then needed to combine an inner solution that resolves the critical layer structure with the outer, normal-mode solution. The result of the asymptotic manipulations is a special reduced system, the 'single-wave model', that describes the particular bifurcation to instability. The 'single wave' refers to the distinct, excited normal mode that occurs in spatially periodic flows. If the flow is 
not geometrically constrained, bands of unstable wavenumbers occur and growing disturbances do not take the form of simple waves. However, spatially periodic jets are more relevant to experiments in circular geometry.

In the current article, we extend the analysis of paper I by considering a special limit of the physical problem. More specifically, linear stability theory identifies a point of neutral stability on the parameter plane where the critical levels (which, in general, occur to either side of the jet axis) merge together along the axis of the jet. The dynamics at this special point is not captured by the theory of paper I, partly because the mean velocity inside the critical layer becomes parabolic, in contrast to the linear shear of the usual critical layer. Here, our goal is to formulate a reduced model that captures the weakly nonlinear dynamics of the merging critical layers. Because we tune the system more finely to arrive at this model, we build in more physics and the dynamics is richer. With the model, we explore the saturation of unstable modes whose critical layers coalesce along the jet axis, as well as a variety of other phenomena also captured by the asymptotic analysis.

From the perspective of nonlinear dynamics and pattern formation, the bifurcation we study is of a higher co-dimension than in paper I. Not only do we capture the bifurcation of an unstable mode from the continuous spectrum, but we also 'unfold' two other bifurcations: the interaction between two discrete, neutral Rossby waves (that leads to a conventional Hamiltonian Hopf bifurcation), and the appearance of a Rossby wave out of the continuum through its edge. Whilst this may be of lesser interest to the fluid dynamicist, it does indicate that the situation is rather special, and aids in deciding which other systems may suffer a similar transition, and that can therefore be described by the same reduced model.

Despite the specific nature of the problem we consider in this article, the results have a more general flavour for two reasons. First, we use the Bickley jet primarily for convenience and continuity with paper I. However, any jet profile can be explored with our methods (there remains a question regarding symmetry about the jet axis that we return to in our conclusions). Second, the reduced model captures more dynamics than the single-wave model of paper I. Therefore, although the model has limited asymptotic validity, it contains more physics and furnishes a useful 'minimal model' of jet dynamics. In particular, in studies of mixing, the reduced model should be the preferred starting point. This was recognized previously by del Castillo-Negrete \& Morrison (1993) whose primary interest was in modelling the transport in experiments with rotating annuli (Solomon et al. 1993). However, their model, which is related to that derived here, lacked the theoretical foundation we provide.

The dynamics of disturbances near the axis of a jet has also been considered by Brunet \& Warn (1990) and Brunet \& Haynes (1995). Their interest focused on how externally introduced perturbations decayed over this special region, and was motivated by simple linear arguments which suggest that perturbations in the streamfunction persist for longer at 'shearless' points. An important difference between the analysis conducted here and those of Brunet \& Warn and Brunet \& Haynes is that we are concerned with an unstable mode that, through its amplification, creates a critical layer along the axis of the jet. In the other studies there was no normal mode and the critical layer had to be suitably forced by an initial disturbance. Nevertheless, there are some common features in the two problems as we uncover later.

A second class of modes with critical levels along the axis of the Bickley jet has also been studied previously (Howard \& Drazin 1964; Mallier \& Davies 1999; Swaters 1999). These 'singular modes' are non-smooth solutions of the inviscid linear problem. By suitably selecting an initial condition, Mallier \& Davies and Swaters 
have considered the evolution of these modes. Because they are not smooth, the singular modes potentially generate more 'nonlinear' behaviour than the smooth modes considered here. However, being linearly stable and often co-existing with smooth, unstable modes, it is difficult to argue for their physical relevance.

\section{Outline of the problem}

\subsection{Formulation}

On the $(x, y)$-plane, the jet can be described by a streamfunction and the vorticity. The dimensionless governing equations are

$$
\begin{aligned}
\omega_{t}+U \omega_{x}+J(\psi, \omega) & =v \nabla^{2} \omega+\left(U^{\prime \prime}-\beta\right) \psi_{x}, \\
\psi_{y y}+\psi_{x x} & =\omega,
\end{aligned}
$$

where $\psi(x, y, t)$ and $\omega(x, y, t)$ denote those parts of the streamfunction and vorticity corresponding to an evolving perturbation and have been separated from the basic flow of the Bickley jet, which is described by the velocity profile, $U(y)=\operatorname{sech}^{2} y$. The parameter $\beta$ measures the size of the variable Coriolis force, and $v$ is a viscosity parameter (an inverse Reynolds number). The nonlinear term is written as $J(\psi, \omega)=$ $\psi_{x} \omega_{y}-\psi_{y} \omega_{x}$.

In $x$, we choose periodic boundary conditions; this allows us to quantize the system and control the number of unstable modes. As we argued in paper I, the precise choice of boundary conditions in $y$ is not especially important because the unstable modes decay exponentially away from the core of the jet, and because we only add viscosity as a perturbation (in which case, viscous effects become important only inside the modal critical layers). For linear stability studies we use either a bounded jet with $-L \leqslant y \leqslant L$ and $\psi(x, \pm L, t)=0$, or an unbounded jet, with $\psi(x, y, t)$ finite as $y \rightarrow \pm \infty$. In the weakly nonlinear analysis, we make use of the unbounded jet, and for numerical computations we use periodic boundary conditions in $y$.

\subsection{Inviscid linear theory}

Linear stability theory proceeds by looking for solutions of the linearized equations of the form expik $(x-c t)$, where $k$ is the (streamwise) wavenumber and $c$ is the (possibly complex) wave speed, or eigenvalue. As discussed in paper I, there are three classes of solutions. First, there is a continuous spectrum that occurs whenever the wave speed of a neutral wave matches the mean flow: $c=c_{r} \in[0,1]$. Second, if $\beta<0$, there may also be Rossby waves (smooth, discrete eigenvalues with $c=c_{r}$ and $c_{r}>1$ ). Third, when $-2 \leqslant \beta \leqslant 2 / 3$, there are complex conjugate pairs over certain ranges of wavenumber $k$, of which one is unstable.

In figure 1, we illustrate the range of unstable modes that correspond to meandering (the even or sinuous modes). The upper boundary of the unstable range locates a smooth neutral mode embedded in the continuous spectrum; in paper I we refered to these as 'inflectional' modes because their critical levels line up with the inflexion points of the mean 'profile', $U(y)-\beta y^{2} / 2$. The distinguishing feature of these modes is that, because of the aligment of their critical levels with the inflexion points, they are smooth eigenmodes.

The lower piece of the stability boundary shown in figure 1 is rather different. The neutral modes lying along this curve are Rossby waves with $c_{r}>1$. Thus, there are two kinds of transitions to instability: in one case, an unstable mode appears by directly bifurcating from the continuous spectrum. In the second, two Rossby waves 
(a)
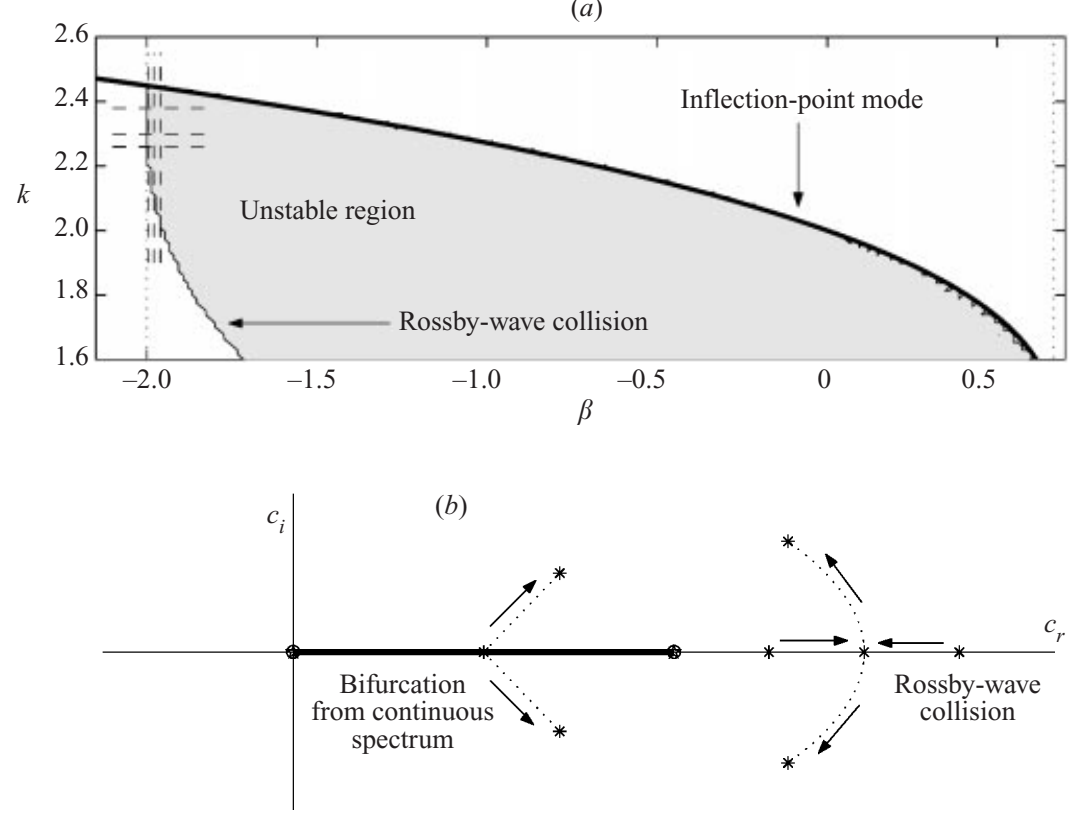

(c)

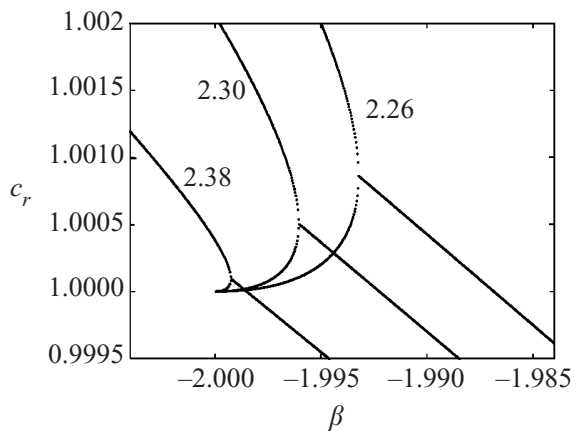

(e)

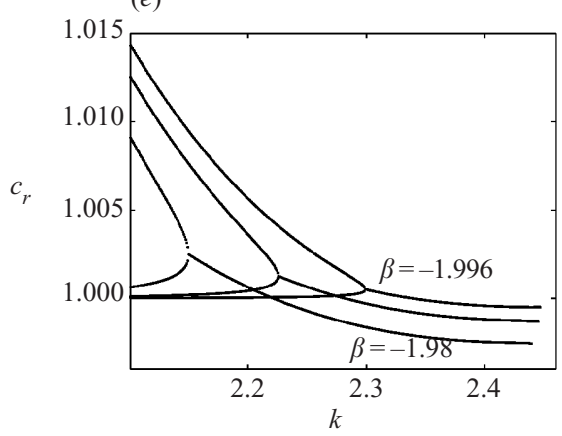

$(d)$

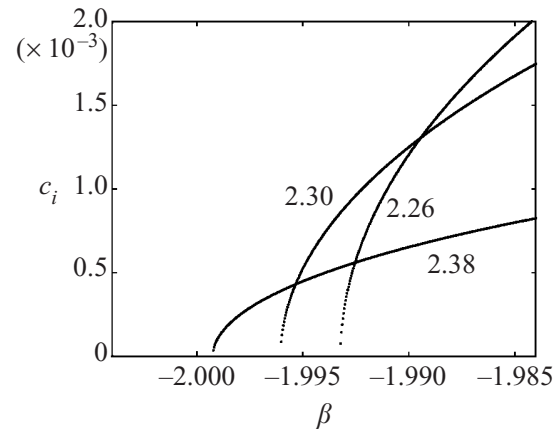

$(f)$

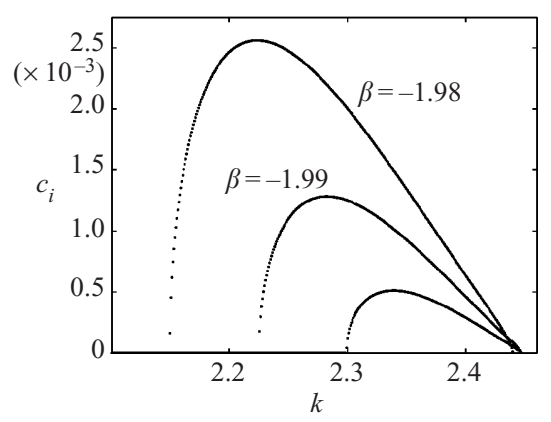

FIGURE 1. (a) Unstable range of wavenumber for the even (sinuous) modes of a bounded Bickley jet in a domain with $L=10$. The thick curve shows an inflectional mode with $\beta=k^{2}\left(4-k^{2}\right) / 6$, which marks the upper stability boundary for an unbounded flow. (b) A sketch of the two possible transitions to instability. $(c, d)$ Numerically determined eigenvalues, $c_{r}$ and $c_{i}$, as functions of $\beta$ for $k=2.26,2.3$ and 2.38. This shows three horizontal cuts through the $(\beta, k)$-parameter plane near the special point, $(\beta, k)=(-2, \sqrt{6}) .(e, f)$ Three vertical cuts, with varying $k$ and $\beta=-1.98,-1.99$ and -1.996 . The dashed lines in $(a)$ show the location of the cuts of $(c-f)$. 
collide in an unstable, resonant, mode interaction (see Drazin, Beaumont \& Coaker 1982). The two pathways to instability are sketched in figure $1(b)$.

The importance of the special point $(\beta, k)=(-2, \sqrt{6})$ is now clear. Here the upper and lower stability boundaries intersect, indicating that both kinds of bifurcations occur simultaneously. The behaviour of the eigenvalues near this special point is illustrated in figure $1(c-f)$. Mode interaction leads to a quadratic dependence of the eigenvalue, $c_{i}$, on the distance to the lower stability boundary. By contrast, when the unstable modes bifurcate out of the continuum on the upper stability boundary, the dependence of $c_{i}$ is linear. Figure 1 also reveals the origin of one of the interacting Rossby waves: as $\beta \rightarrow-2$, for each of the values of $k$, the mode with lower wave speed disappears into the continuous spectrum through $c=(1,0)$. This trend was previously noted by Drazin et al. (1982).

In summary, the cuts shown in figure 1 illustrate the important facets of the eigenvalue problem in the vicinity of the special point, $(-2, \sqrt{6})$. Here, three bifurcations occur at once: the bifurcation of a conjugate pair out of the continuous spectrum, the splitting of this pair into two real modes, and the disappearance of one of these real modes back into the continuum through the branch point at $c=1$.

\section{Critical layers along the axis of the jet}

The expansion carried out in paper I is designed to capture the nonlinear dynamics of a mode coupled to two separated critical layers. The model cannot therefore capture the behaviour near the point $(\beta, k)=(-2, \sqrt{6})$, where the two critical layers merge together, and we must develop an alternative reduced model. To this end, we again construct an asymptotic solution to the governing equations. We begin by transforming into a frame moving with the maximal speed of the jet; this is equivalent to taking a slightly different basic velocity profile, $U=\operatorname{sech}^{2} y-1$. Then, the expansion proceeds with a new set of scalings of the parameters and asymptotic sequences. Because we develop an expansion that unfolds the region on the parameter plane surrounding a special point, rather than a curve, we require two control parameters, namely $\beta$ and $k$. The new scalings take the form

$$
\partial_{t} \rightarrow \epsilon^{2} \partial_{T}, \quad k^{2} \rightarrow 6+\epsilon \mu, \quad \beta \rightarrow-2+\epsilon^{2} \beta_{2}, \quad v \rightarrow \epsilon^{4} v_{4}
$$

and $(\psi, \omega) \sim \epsilon^{3}$. These scalings are motivated by the results for the linear problem (that is, the structure of the eigenvalues near the special point) and the need to take a distinguished limit in which instability, nonlinearity and viscosity appear in the reduced model simultaneously.

With the frame shift and scalings of (3.1), the governing equations become

$$
\begin{gathered}
\epsilon^{2} \omega_{T}-\omega_{x} \tanh ^{2} y+J(\psi, \omega)=\epsilon^{4} v_{4} \nabla^{2} \omega+2 \psi_{x} \tanh ^{2} y\left(1+3 \operatorname{sech}^{2} y\right)-\epsilon^{2} \beta_{2} \psi_{x}, \\
\psi_{y y}+\psi_{x x}=\omega .
\end{gathered}
$$

To deal with the change in domain size, we Fourier transform (in $x$ ) the Poisson relation:

$$
\hat{\omega}(n, y, T)=\partial_{y}^{2} \hat{\psi}(n, y, T)-n^{2}(6+\epsilon \mu) \hat{\psi}(n, y, T),
$$

where the hat denotes the Fourier transform, $n$ is the discrete Fourier mode index, and we have used the fact that the domain size is selected so that the minimum wavenumber is $k$.

We solve the equations using matched asymptotics. We first develop a regular expansion and then demonstrate how the regular solution breaks down in an inner, 
critical layer surrounding the axis of the jet. Inside the critical layer, we find another solution that removes the apparent singularity. Finally we match the regular (outer) and inner solutions.

\subsection{Regular expansion}

We introduce the sequences

$$
\psi=\epsilon^{3} \psi_{3}+\epsilon^{4} \psi_{4}+\cdots, \quad \omega=\epsilon^{3} \omega_{3}+\epsilon^{4} \omega_{4}+\cdots .
$$

At lowest order,

$$
\tanh ^{2} y\left[\omega_{3}+2\left(1+3 \operatorname{sech}^{2} y\right) \psi_{3}\right]=0, \quad \omega_{3}=\psi_{3 y y}+\psi_{3 x x},
$$

which has a neutral inflectional mode as the solution:

$$
\begin{gathered}
\psi_{3}=A(T) \mathrm{e}^{\mathrm{i} k x} \operatorname{sech}^{2} y+\text { c.c., } \\
\omega_{3}=-2 \operatorname{sech}^{2} y\left(1+3 \operatorname{sech}^{2} y\right) A(T) \mathrm{e}^{\mathrm{i} k x}+\text { c.c. }
\end{gathered}
$$

At $O\left(\epsilon^{4}\right)$, we find

$$
\tanh ^{2} y\left[\omega_{4}+2\left(1+3 \operatorname{sech}^{2} y\right) \psi_{4}\right]=0, \quad \hat{\omega}_{4}=\hat{\psi}_{4 y y}-6 n^{2} \hat{\psi}_{4}-\mu A \delta_{n 1} \operatorname{sech}^{2} y,
$$

where $\delta_{i j}$ is the Kronecker delta. At this order, it becomes necessary to allow for an arbitrary jump in the streamwise velocity component, $\psi_{y}$, which is equivalent to accounting for a line source of vorticity along the jet's axis. One could also argue that this jump is necessary to account for the singular operation of dividing by $\tanh ^{2} y$ in the first expression in (3.9). We then write

$$
\hat{\psi}_{4 y y}+\left(2-6 n^{2}+6 \operatorname{sech}^{2} y\right) \hat{\psi}_{4}=\mu A \delta_{n 1} \operatorname{sech}^{2} y+\hat{C}(n, T) \delta(y) .
$$

The delta-function term, $C(x, T) \delta(y)$, is a formal device used to represent the arbitrary jump in $\psi_{4 y}$ across $y=0$. In order to ensure that this equation has a solution, we multiply by $\operatorname{sech}^{2} y$ and integrate in $y$, omitting a small region, $[-\varsigma, \varsigma]$ with $\epsilon \ll \varsigma \ll 1$, surrounding $y=0$. Then,

$$
\hat{C}(1, T) \equiv\left[\hat{\psi}_{4 y}\right]_{-\varsigma}^{\varsigma}=-\frac{4}{3} \mu A,
$$

to order $\varsigma$.

At $O\left(\epsilon^{5}\right)$,

$$
\tanh ^{2} y\left[\omega_{5 x}+2\left(1+3 \operatorname{sech}^{2} y\right) \psi_{5 x}\right]=\omega_{3 T}+\beta_{2} \psi_{3 x} .
$$

This indicates that $\omega_{5}$ diverges as $y \rightarrow 0$, and is where the critical-level singularity visibly enters the expansion.

We summarize the expansion so far by quoting the limits of the outer solution for $1 \gg y \gg \epsilon$ :

$$
\begin{gathered}
\hat{\psi}(1, y, T)=\epsilon^{3}\left(1-y^{2}\right) A+\frac{1}{2} \epsilon^{4}\left[\hat{C}(1, T)|y|+\mu y^{2} A\right]+\epsilon^{5}\left(\frac{8 A_{T}}{\mathrm{i} k_{c}}-\beta_{2} A\right) \log y+O\left(\epsilon^{3} y^{4}\right), \\
\hat{\psi}_{y}(1, y, T)=-2 \epsilon^{3} y A+\epsilon^{4}\left[\mu y A \pm \frac{1}{2} \hat{C}(1, T)\right]+\epsilon^{5}\left(\frac{8 A_{T}}{\mathrm{i} k_{c}}-\beta_{2} A\right) \frac{1}{y}+O\left(\epsilon^{3} y\right), \\
\hat{\omega}(1, y, T)=-8 \epsilon^{3} A-\epsilon^{5}\left(\frac{8 A_{T}}{\mathrm{i} k_{c}}-\beta_{2} A\right) \frac{1}{y^{2}}+O\left(\epsilon^{3} y^{2}\right) .
\end{gathered}
$$

On comparing the various term in the asymptotic sequence, we discover the scalings of the inner solution: terms first break order in $\hat{\psi}_{y}$ and $\hat{\omega}$ when $y \sim \epsilon$. 
3.2. Inner solution

For the inner solution, we introduce the scalings and sequences

$$
\begin{gathered}
y=\epsilon Y, \quad \partial_{y} \rightarrow \epsilon^{-1} \partial_{Y}, \\
\psi=\epsilon^{3} \Psi_{3}+\epsilon^{4} \Psi_{4}+\epsilon^{5} \log \epsilon \Upsilon_{5}+\epsilon^{5} \Psi_{5}+\cdots, \quad \omega=\epsilon^{3} Z ;
\end{gathered}
$$

the logarithmic terms in the sequence are standard in critical-layer expansions and are required because of the inner limit of the outer solution evident in (3.13)

We deal with the Poisson relation first: in terms of the inner variables,

$$
Z=\frac{1}{\epsilon^{2}} \Psi_{Y Y}+\Psi_{x x}
$$

Thus,

We take

$$
\Psi_{3 Y Y}=\Psi_{4 Y Y}=\Upsilon_{5 Y Y}=0 .
$$

$$
\Psi_{3}=A \mathrm{e}^{\mathrm{i} k_{c} x}+\text { c.c., } \quad \Psi_{4}=0, \quad \Upsilon_{5}=\left(\frac{8 A_{T}}{\mathrm{i} k_{c}}-\beta_{2} A\right) \mathrm{e}^{\mathrm{i} k_{c} x}+\text { c.c. },
$$

which ensure a match of the streamfunction to order $\epsilon^{5} \log \epsilon$.

At order $\epsilon^{3}$,

$$
\Psi_{5 Y Y}=Z-\Psi_{3 x x},
$$

which provides the integral relation,

$$
\left[\Psi_{5 Y}\right]_{-\Delta}^{\Delta}=\int_{-\Delta}^{\Delta} Z \mathrm{~d} Y+12 \Delta \Psi_{3}
$$

To leading order, the vorticity equation becomes

$$
Z_{T}-Y^{2} Z_{x}+\Psi_{3 x} Z_{Y}=v_{4} Z_{Y Y}+8 Y^{2} \Psi_{3 x}-\beta_{2} \Psi_{3 x} .
$$

This relation indicates that $Z \sim-8 \Psi_{3}$ as $Y \rightarrow \pm \infty$, which matches onto the leadingorder term in (3.15). We now introduce a new, localized vorticity variable, $\zeta(x, Y, T)=$ $Z+8 \Psi_{3}$, in terms of which (3.23) becomes

$$
\zeta_{T}-Y^{2} \zeta_{x}+\Psi_{3 x} \zeta_{Y}=v_{4} \zeta_{Y Y}+8 \Psi_{3 T}-\beta_{2} \Psi_{3 x}
$$

The new variable has the far-field form, $\zeta \sim Y^{-2}\left[\beta_{2} A+8 \mathrm{i} A_{T} / k_{c}\right] \mathrm{e}^{\mathrm{i} k_{c} x}+$ c.c., which matches the second-order term in (3.15). However, in general, (3.24) must be solved as a nonlinear partial differential equation.

To complete the formulation of the reduced model, we must connect $A(T)$ and $\zeta$, which is accomplished by matching (3.22) with the corresponding jump from (3.14). Let $y \rightarrow \varsigma=\epsilon \Delta$ and $Y \rightarrow \pm \Delta$ denote the outer and inner coordinates in an intermediate matching region, where $\epsilon^{-1} \gg \Delta \gg 1$. Then, the outer limit of the inner solution is

$$
\epsilon^{4}\left[\Psi_{5 Y}\right]_{-\Delta}^{\Delta}=\epsilon^{4} \int_{-\Delta}^{\Delta} \zeta \mathrm{d} Y-4 \epsilon^{4} \Delta \Psi_{3}
$$

(which includes an extra factor of $\epsilon$ to account for the change of variable, $y \rightarrow Y$ ). On the other hand, the inner limit of the outer solution becomes

$$
\left[\psi_{y}\right]_{-\epsilon \Delta}^{\epsilon \Delta} \sim-4 \epsilon^{4} \Delta\left(A \mathrm{e}^{\mathrm{i} k_{c} x}+\text { c.c. }\right)+\epsilon^{4} C+2 \frac{\epsilon^{4}}{\Delta}\left[\left(\frac{8 A}{\mathrm{i} k_{c}}-\beta_{2} A\right) \mathrm{e}^{\mathrm{i} k_{c} x}+\text { c.c. }\right] .
$$


On equating the two, exploiting (3.11), and formally taking $\Delta \rightarrow \infty$, we find

$$
-\frac{4}{3} \mu A=\frac{k_{c}}{2 \pi} \int_{-\pi / k_{c}}^{\pi / k_{c}} \mathrm{~d} x \int_{-\infty}^{\infty} \mathrm{d} Y \mathrm{e}^{-\mathrm{i} k_{c} x} \zeta \equiv\left\langle e^{-\mathrm{i} k_{c} x} \zeta\right\rangle .
$$

Equations (3.24) and (3.27) form the reduced asymptotic model.

\subsection{Canonical form}

We place equations (3.24), (3.27) into a more convenient form by rescaling. We adopt

$$
\zeta=\frac{4}{3} \mu \varpi^{2} \zeta^{\prime}, \quad A^{*}=-\varpi^{3} A^{\prime}, \quad k_{c} x=-x^{\prime}, \quad \varpi Y=Y^{\prime}, \quad \varpi^{2} k_{c} T=T^{\prime},
$$

where $\varpi$ is a positive free parameter. On discarding the primes, this brings the reduced equations into their final form,

$$
\begin{gathered}
\zeta_{T}+Y^{2} \zeta_{x}+\varphi_{x} \zeta_{Y}=\lambda \zeta_{Y Y}-\kappa \varphi_{T}-\gamma \varphi_{x} \\
A=\left\langle\mathrm{e}^{-\mathrm{i} x} \zeta\right\rangle \frac{1}{2 \pi} \int_{-\pi}^{\pi} \mathrm{d} x \int_{-\infty}^{\infty} \mathrm{d} Y \mathrm{e}^{-\mathrm{i} x} \zeta, \quad \varphi=A \mathrm{e}^{\mathrm{i} x}+\text { c.c. }
\end{gathered}
$$

where

$$
\lambda=\frac{v_{4}}{k_{c} \varpi^{4}}, \quad \kappa=\frac{6 \varpi}{\mu}, \quad \gamma=\frac{3 \beta_{2}}{4 \mu \varpi} .
$$

Finally, we may select $\varpi$ to scale one of the parameters, $\kappa$ and $\gamma$, to unity in absolute value. In numerical computations, we choose $\varpi$ so that $\gamma= \pm 1$. (Then, $\kappa=9 \beta_{2} / 2 \mu^{2} \operatorname{sgn}\left(\beta_{2} \mu\right)$.)

\section{Conservation laws, initial conditions and symmetries}

The reduced model provides a powerful tool with which we can explore criticallayer dynamics along the axis of the jet. In this section, we begin our exploration of the model.

The model has a number of integral relations that it inherits from the original equations:

$$
\begin{gathered}
\langle\zeta\rangle_{T}=0, \quad\langle Y \zeta\rangle_{T}=0, \quad \frac{1}{2}\left\langle\zeta^{2}\right\rangle_{T}+\kappa|A|_{T}^{2}=-\lambda\left\langle\zeta_{Y}^{2}\right\rangle, \\
\frac{1}{3}\left\langle Y^{3} \zeta-6 \kappa|A|^{2}\right\rangle_{T}-|A|_{T}^{2}=2 \lambda\langle Y \zeta\rangle,
\end{gathered}
$$

which correspond to conservation of vorticity and momentum in the $x$-direction, and the balance of enstrophy and energy, respectively. The energy integral assumes that $\zeta$ has the far-field form

$$
\zeta \sim \frac{\kappa \varphi_{T x}-\gamma \varphi}{Y^{2}}+\frac{2 \kappa|A|^{2}}{Y^{3}}
$$

To ensure convergence in the remaining expressions, we always evaluate the integral in $x$ first. If $\lambda=0$, the first of these relations can be expanded into $\langle F(q)\rangle_{T}=0$, for any function, $F(q)$, of the total vorticity,

$$
q=\zeta+\gamma Y+\kappa\left(\varphi-Y^{3} / 3\right)
$$

which corresponds to the usual infinitude of Casimir invariants for an inviscid fluid.

Because our interest surrounds the dynamics of unstable modes, the best choice for the initial condition is the shape of the relevant normal mode. However, as we see below, normal modes do not always exist for all values of the parameters. 
A compromise is

$$
\zeta(x, Y, T=0)=\frac{2 A_{0} \cos x}{\pi\left(1+Y^{2}\right)}, \quad A(T=0)=A_{0},
$$

where $A_{0}$ is a small initial amplitude that we typically take to be $10^{-2}$. The initial vorticity distribution is then localized within the critical region, with a form similar to any normal mode (see $\S 4.2$ ), and additionally excites a small, shearing, vorticity rearrangment (see $\S 4.3$ ).

Given the initial condition in (4.5), the system is invariant under the transformation,

$$
x \rightarrow \pi+x, \quad Y \rightarrow-Y, \quad \zeta \rightarrow-\zeta, \quad A \rightarrow A, \quad \varphi \rightarrow-\varphi .
$$

This symmetry is also preserved in numerical solutions of both the reduced model and the full equations, suggesting that solutions with the symmetry are stable. Consequently, we exploit this symmetry in most of the numerical computations of the reduced model to double the resolution. This also means that vorticity conservation, $\langle\zeta\rangle=0$, is satisfied exactly for all time.

In order to perform computations with the reduced model, we rewrite the vorticity equation in the form

$$
q_{T}+Y^{2} q_{x}+\varphi_{x} q_{Y}=\lambda\left(q+\kappa Y^{3} / 3\right)_{Y Y},
$$

and then use the operator splitting scheme described in paper I and Balmforth, Llewellyn Smith \& Young (2000). (We choose a domain of size $[-6,6]$ in $Y$ for most of the computations, and typically use 256 gridpoints in $x$ and 1025 in $Y$.)

\section{Linear theory of the reduced model}

We now revisit the linear stability problem using the reduced model and recover the key features of the numerically generated eigenvalues near $(\beta, k)=(-2, \sqrt{6})$. This is also an essential exercise in understanding the dynamics of the model. We start from the linearized equations, projected onto the Fourier mode $\mathrm{e}^{\mathrm{i} x}: \zeta=\hat{\zeta} \mathrm{e}^{\mathrm{i} x}+$ c.c., where

$$
\hat{\zeta}_{T}+\mathrm{i} Y^{2} \hat{\zeta}^{-}-\nu \hat{\zeta}_{Y Y}=-\kappa A_{T}-\mathrm{i} \gamma A, \quad A=\int_{-\infty}^{\infty} \hat{\zeta} \mathrm{d} Y
$$

\subsection{Inviscid normal modes}

The inviscid normal modes, with dependence $\mathrm{e}^{\mathrm{i}\left(x-c_{2} T\right)}$, satisfy

$$
\hat{\zeta}=\frac{c_{2} \kappa-\gamma}{Y^{2}-c_{2}} \mathrm{e}^{-\mathrm{i} c_{2} T} \text {. }
$$

Hence,

$$
\mathscr{D}\left(c_{2}\right)=1-\left(\kappa c_{2}-\gamma\right) \int_{-\infty}^{\infty} \frac{\mathrm{d} Y}{Y^{2}-c_{2}}=0,
$$

defines the dispersion relation. Let $c_{2}=\lambda^{2} \equiv\left(\lambda_{r}+\mathrm{i} \lambda_{i}\right)^{2}$. Then,

$$
\mathrm{i} \pi\left(\kappa \lambda^{2}-\gamma\right)-\lambda \operatorname{sgn}\left(\lambda_{i}\right)=0 .
$$

There are two possible sets of solutions to this equation:

$$
\begin{aligned}
& \lambda_{r}= \pm \sqrt{\frac{\left(4 \pi^{2} \kappa \gamma-1\right)}{4 \pi^{2} \kappa^{2}}}, \quad\left|\lambda_{i}\right|=-\frac{1}{2 \pi \kappa} ; \\
& \lambda_{r}=0, \quad\left|\lambda_{i}\right|=\frac{1}{2 \pi \kappa}\left[-1 \pm \sqrt{1-4 \pi^{2} \kappa \gamma}\right] .
\end{aligned}
$$



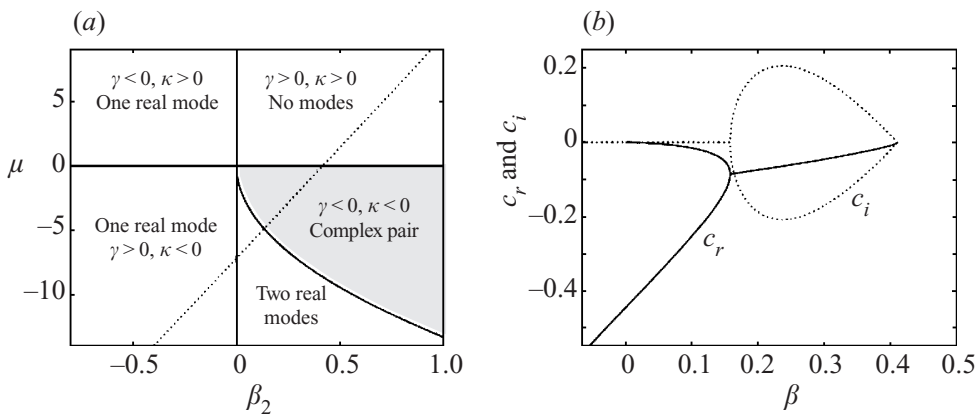

FiguRE 2. (a) Regime diagram for normal modes. The shaded area corresponds to the unstable region. (b) A sample set of eigenvalues along the cut shown by the dotted line in $(a)$.

The solutions (i) correspond to complex conjugates; the pair (ii) are real modes. Because of the absolute values and square roots that appear in the formulae, the solutions do not always exist. In fact, their existence depends on the two parameters $\kappa$ and $\gamma$, and particularly their signs. A sketch that summarizes the possibilities is given in figure 2, which should be compared with figure 1.

The complex-conjugate mode pair has the eigenvalue

$$
c_{2 r}=\frac{\gamma}{\kappa}-\frac{1}{2 \pi^{2} \kappa^{2}}, \quad c_{2 i}= \pm \frac{1}{2 \pi^{2} \kappa^{2}} \sqrt{4 \pi^{2} \kappa \gamma-1} .
$$

In the asymptotic theory, the collision of Rossby waves therefore occurs along the curve $4 \pi^{2} \kappa \gamma=1$ for $\kappa<0$. In the original variables, this curve is $\mu=-\pi \sqrt{18 \beta_{2}}$. The bifurcation from the continuous spectrum, on the other hand, occurs when $\kappa^{-1}$ passes through zero for $\gamma / \kappa$ finite and positive. Then, $\left|c_{2 i}\right| \rightarrow 0$ and $c_{2 r} \rightarrow \gamma / \kappa$. (If $\gamma$ remains finite as $\kappa \rightarrow-\infty$, the complex modes disappear into the continuous spectrum through the branch point at the origin.) Equivalently, this bifurcation occurs along $\mu=0$ for $\beta_{2}>0$ and $\beta_{2} / \mu^{2}$ finite.

Lastly, it is important to note that the dispersion function, $\mathscr{D}\left(c_{2}\right)$, is non-analytic because the integral over $Y$ is singular along the positive real axis. This line is where the vorticity fluctuation (5.2) contains singular points, or critical levels, and therefore locates the continuous spectrum. To make the dispersion function single-valued on the complex plane, we introduce a branch cut along the positive real axis, in which case

$$
\mathscr{D}\left(c_{2}\right)=1-\frac{\mathrm{i} \pi}{\sqrt{c_{2}}}\left(\kappa c_{2}-\gamma\right) \quad \text { with } \quad 0<\arg \left(c_{2}\right) \leqslant 2 \pi,
$$

which is equivalent to (5.4). Note that the inconsistent solutions to (5.5)-(5.6), obtained by ignoring the absolute values, correspond to zeros of the dispersion function on different Riemann sheets.

\subsection{Inviscid initial-value problem}

We next consider the linear initial-value problem. A Laplace transform of (5.1) with initial condition (4.5) leads to

$$
\tilde{\zeta}=\frac{A_{0} /\left[\pi\left(1+Y^{2}\right)\right]+\kappa A_{0}-(\kappa s+\mathrm{i} \gamma) \tilde{A}}{s+\mathrm{i} Y^{2}}, \quad \tilde{A}=A_{0} \frac{\pi \kappa+(1-\mathrm{i} \sqrt{\mathrm{is}})^{-1}}{\pi \kappa s+\mathrm{i} \pi \gamma+\sqrt{\mathrm{is}}},
$$

where the tilde denotes the transformed variable, with coordinate $s$. To derive these formulae, we evaluate the integrals in $Y$ assuming that $s$ is in the right half-plane, 
(a)

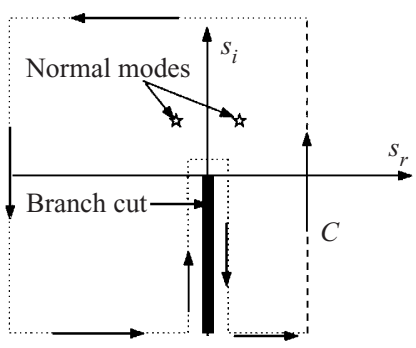

(b)

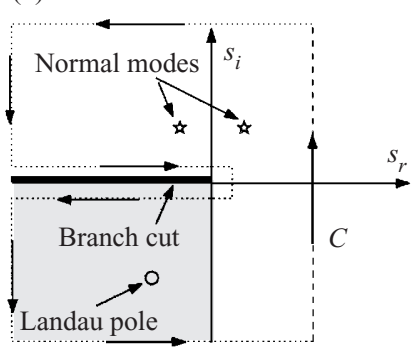

FIGURE 3. The Bromwich contour $C$ and the sections added to it to create a closed contour. In $(a)$ the branch cut is chosen to lie along the 'spectral' cut, the negative imaginary axis. The contour then encloses only normal modes. In $(b)$, the cut is placed along the negative real axis, thereby allowing the uncovering of Landau poles or quasi-modes by analytic continuation of the dispersion relation (and the covering of normal modes in the third quadrant).

which follows if $s$ lies along the path of the inverse Laplace transform, the Bromwich contour (a vertical line lying to the right of any singularities of $\tilde{A}(s)$ ). These singularities occur at the zeros of the denominator, which corresponds to the dispersion function (5.8), and from any poles in the numerator. The detection of the latter, together with the computation of the inverse transform as a whole, forces us to deal with the non-analyticity in (5.9).

Two options are open to us for the position of the branch cut associated with the square root in $\tilde{A}(s)$. First, we may position the cut along the negative imaginary axis, as shown in figure $3(a)$. In this case, the cut lines up with the 'spectral' cut of the continuous spectrum and the dispersion function (5.8), and is arguably the most physical $\left(s \equiv-\mathrm{i} c_{2}=-\mathrm{i} Y^{2}\right.$ for any real $\left.Y\right)$. However, a second cut, taken along the negative real axis (see figure $3 b$ ) is more convenient in uncovering the long-time behaviour of the mode amplitude, as explained below. The key difference between the two cases is that one must analytically continue the denominator of $\tilde{A}(s)$ (the dispersion function) into the third quadrant in the second case; that is, through the spectral cut and onto a new Riemann sheet. Equivalently, we look for zeros of $\mathscr{D}($ is) with $-\pi / 2<\arg ($ is $) \leqslant 3 \pi / 2$, in contrast to (5.8). The continuation can therefore pick out zeros of the continued dispersion function that do not correspond to normal modes, and any true normal mode in the third quadrant does not contribute. The procedure is equivalent to the uncovering of Landau damping in the Vlasov equation; the new poles, or 'fake' eigenvalues are 'Landau poles' or 'quasi-modes'.

With the branch cut along the negative portion of the real axis, the inverse transform can be evaluated using a contour integral on the cut plane. To the Bromwich contour, we add an arc enclosing the entire left half-plane and two sections that surround the branch cut; see figure $3(b)$. The combined contour integral must then equal the sum of the residues of any poles that lie in the enclosed region; these poles correspond to any normal modes in the upper half-plane or fourth quadrant, and any quasi-modes in the third quadrant.

We may now write

$$
A(T)=A_{\mathrm{p}}(T)+A_{\mathrm{bc}}(T)
$$

where

$$
A_{\mathrm{p}}(T)=2 A_{0}\left(\kappa+\gamma+\pi^{-1}\right) \sum_{j} \frac{s_{j} \mathrm{e}^{s_{j} T}}{\left(\kappa s_{j}-\mathrm{i} \gamma\right)\left(1+\mathrm{i} s_{j}\right)}
$$


denotes the residues of the poles located at $s=s_{j}$ (the precise number of which depends on $\kappa$ and $\gamma$ ), and the contribution of the branch cut is

$$
A_{\mathrm{bc}}(T)=-2 A_{0} \sqrt{\mathrm{i}}\left(\kappa+\gamma+\pi^{-1}\right) \int_{0}^{\infty} \frac{\chi^{2} \mathrm{e}^{-\chi^{2} T} \mathrm{~d} \chi}{\pi^{2}\left(\gamma+\mathrm{i} \kappa \chi^{2}\right)^{2}-\mathrm{i} \chi^{2}},
$$

which can be written in terms of error functions. For $T \gg 1$,

$$
A_{\mathrm{bc}}(T) \sim-\frac{A_{0} \sqrt{\mathrm{i}}}{2 \gamma^{2}}\left(\kappa+\gamma+\pi^{-1}\right)(\pi T)^{-3 / 2},
$$

which has the same form as the contribution found by Brunet \& Haynes (1995) for a balanced parabolic jet, and differs from that expected for monotonic shear flows (which is generically $t^{-2}-$ Brown \& Stewartson 1980).

The combination of the algebraic decay in (5.13) and the exponential decay of any quasi-modes reflects 'continuum damping', the phase mixing of perturbations within the continuous spectrum. The decay is only a property of the streamfunction: a parallel analysis for the vorticity fluctuation $\tilde{\zeta}(s)$ always produces non-decaying terms with dependence $\mathrm{e}^{-\mathrm{i} Y^{2} T}$ whenever there is continuum damping, which represents the tilting and filamentation of the initial perturbation by the background shear. The mode amplitude $A(T)$ decays because this variable is an integral average of the increasingly crenellated vorticity.

\subsection{Viscous normal modes}

To find the viscous normal modes we Fourier transform in $Y$ :

$$
\check{\zeta}_{p p}+\left(c_{2}+\mathrm{i} \lambda p^{2}\right) \check{\zeta}=-2 \pi\left(\kappa c_{2}-\gamma\right) A \delta(p),
$$

where $\check{\zeta}(p)$ refers to the transform of $\hat{\zeta}(Y)$, and $p$ is the transformed coordinate. The solution to this equation can be written in terms of parabolic cylinder functions:

$$
\check{\zeta}(p)=-\frac{\pi}{\alpha}\left(\kappa c_{2}-\gamma\right) A \frac{U(b, \alpha|p|)}{U^{\prime}(b, 0)}, \quad \alpha=(-4 \mathrm{i} \lambda)^{1 / 4}, \quad b=-\frac{c_{2}}{\alpha^{2}},
$$

where $U(a, x)$ is the parabolic cylinder function, as defined in Abramowitz \& Stegun (1972, section 19). Because $\breve{\zeta}(p=0) \equiv \int_{-\infty}^{\infty} \hat{\zeta}(Y) \mathrm{d} Y$, the dispersion relation then follows:

$$
\mathscr{D}\left(c_{2}\right)=1+\frac{\pi}{\alpha}\left(\kappa c_{2}-\gamma\right) \frac{U(b, 0)}{U^{\prime}(b, 0)} \equiv 1-\frac{\pi}{\alpha \sqrt{2}}\left(\kappa c_{2}-\gamma\right) \frac{\Gamma(b / 2+1 / 4)}{\Gamma(b / 2+3 / 4)}=0,
$$

where $\Gamma(x)$ is the Gamma-function.

To connect this dispersion relation with the inviscid version, we take the limit $\lambda \rightarrow 0$, or $|b| \rightarrow \infty$. By making use of Stirling's formula, we find

$$
0=\mathscr{D}\left(c_{2}\right) \sim 1-\frac{\mathrm{i} \pi}{\sqrt{c_{2}}}\left(\kappa c_{2}-\gamma\right), \quad-\pi / 4<\arg \left(c_{2}\right) \leqslant 7 \pi / 4
$$

This is identical to the inviscid dispersion relation found earlier, except for a difference in the range of the argument of $c_{2}$. Thus, the inviscid normal modes are the limits of the viscous modes as $\lambda \rightarrow 0$, where the two ranges coincide $\left(0<\arg \left(c_{2}\right) \leqslant 7 \pi / 4\right)$. However, the inviscid eigenvalues with $\arg \left(c_{2}\right)>7 \pi / 4$ are not the limits of viscous modes, and inviscid quasi-modes in $0<\arg \left(c_{2}\right)<-\pi / 4$ are limiting viscous eigenvalues. This correspondence, with some twists, parallels what happens for the single-wave model of paper I and in related systems (Balmforth 1998). 
In the opposite limit of large viscosity, $\lambda \gg 1$, there is one obvious asymptotic balance, namely

$$
c_{2} \approx \frac{2 \lambda^{1 / 4} \mathrm{e}^{-\mathrm{i} \pi / 8} \Gamma(3 / 4)}{\pi \kappa \Gamma(1 / 4)} .
$$

Thus, modal stability depends only on the sign of $\kappa: \kappa<0$ (or $k^{2}<6$ ) implies instability. Though this confirms that viscous instabilities occur inside the unstable region of inviscid theory, the prediction of instability for $\gamma>0(\beta<-2)$ is surprising. We interpret this result as the viscous destabilization of an otherwise neutral Rossby wave. This interpretation is confirmed by numerically solving the viscous eigenvalue problem for periodic jets: the stability boundary is shifted to $\beta<-2$ on adding viscosity. The asymptotic scheme only captures the shift of the boundary from $\beta=-2$, and not its new location. In practice, for reasons uncovered in the next section and in the Appendix, we limit ourselves to consideration of cases in which $\lambda$ remains small. Viscous destabilization is then a secondary issue that we ignore.

In addition to the modes detected above, there is also an infinite set of discrete modes that are approximately distributed along the line $\arg \left(c_{2}\right)=-\pi / 4$. More precisely, when $\left|\left(\kappa c_{2}-\gamma\right) / \sqrt{\alpha}\right| \gg 1$, we must balance the diverging term in the dispersion function by suitably arranging $3 / 4+b / 2$ to lie near a pole of $\Gamma(3 / 4+b / 2)$. Then,

$$
c_{2} \approx(2 n+3 / 2) \sqrt{\lambda} \mathrm{e}^{-\mathrm{i} \pi / 4}, \quad n=0,1,2 \ldots .
$$

In the limit $\lambda \rightarrow 0$, this set of eigenvalues becomes increasingly dense and limits to a continuous distribution. However, this distribution is not the same as the inviscid continuum.

The results of this section illustrate some of the peculiar features of shear flow stablity theory and the curious connections and distinctions between the inviscid and viscous problems.

\section{Nonlinear dynamics}

\subsection{Stable jets}

According to the inviscid linear theory presented above, except in the shaded region of figure 2, perturbations to the jet are stable, and either generate neutral Rossby waves or decay by continuum damping. We confirm these predictions by numerically solving the reduced, nonlinear model equations (3.29)-(3.30).

Two cases with neutral Rossby waves are shown in figure 4; these correspond to jets in the second and third quadrants of figure 2. The initial disturbance sets the Rossby waves 'ringing'. As shown by the snapshots of the vorticity field, these waves correspond to propagating meanders of the jet (there is also some residual filamentation as part of the initial vortical disturbance is tilted over and sheared out). The numerical solution agrees with the linear theory up to a slight difference in the wave speed which arises chiefly because the numerical computation is performed on a finite domain. These computations are performed with $\lambda=0$, which signifies that the code runs with an implicit dissipation connected to finite resolution. This implicit dissipation roughly corresponds to an explicit viscosity of about $10^{-6}$ (depending on the precise resolution) and, though small, can appreciably affect long-time computations. For the shorter runs in figure 4, however, it is negligible.

In figure 5 we show a jet with parameter values from the first quadrant of figure 2 (again $\lambda=0$ ). In this case, there are no Rossby waves and, as predicted by linear theory, the perturbation decays by continuum damping. Moreover, there is a clear 

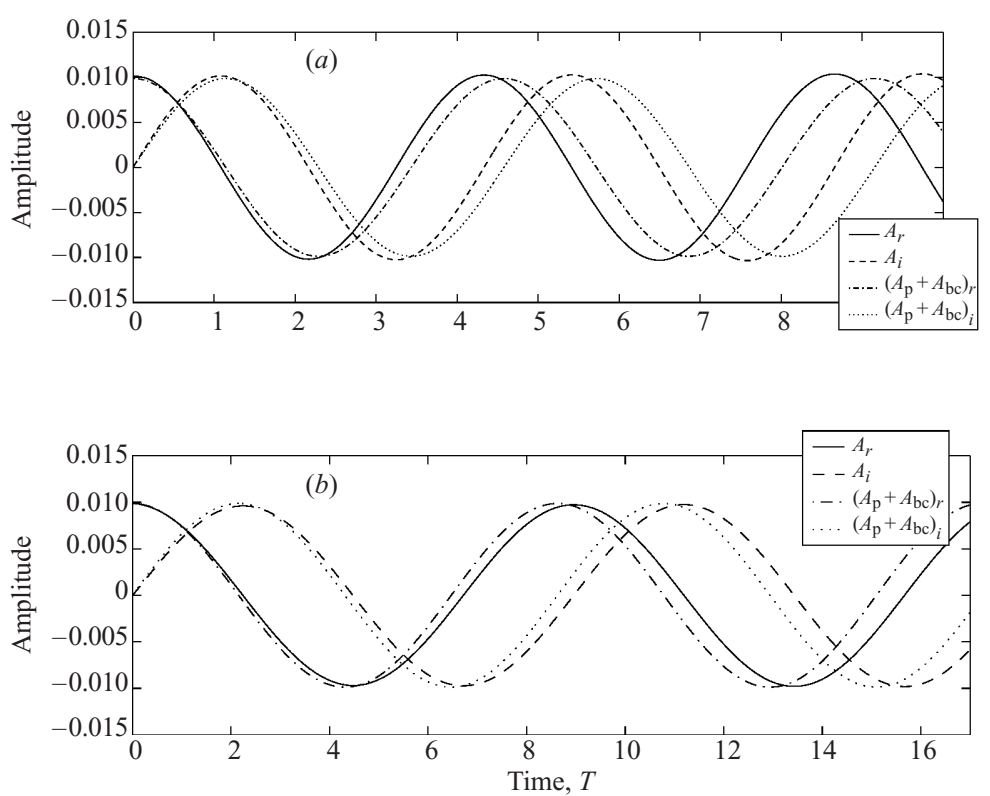

(c)

$(d)$
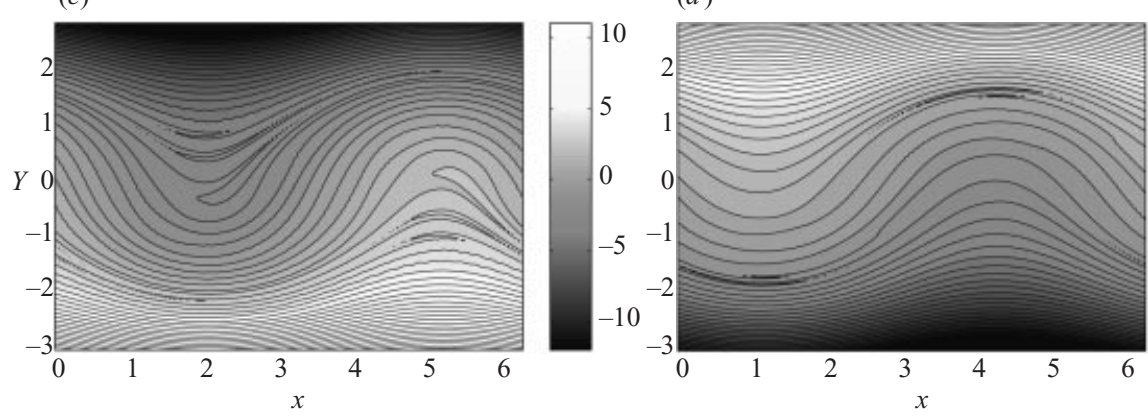

FiguRE 4. Stable jets with $(a) \kappa=-1$ and $\gamma=1$ at $T=20$, and $(b) \kappa=1$ and $\gamma=-1$ at $T=28$. Both the numerical solution (with $\lambda=0$ ) and $A_{p}+A_{b c}$ are shown. $(c, d)$ Corresponding snapshots of the total vorticity field, $q=\zeta+\gamma Y+\kappa\left(\varphi-Y^{3} / 3\right)$, on the $(x, Y)$-plane, with shading as given by the keys. In $(a, b)$, the initial disturbance has amplitude $A_{0}=0.01$. In $(c, d)$, the meander is emphasized by taking $A_{0}=1$. Despite this large change in the initial amplitude, the oscillations of the mode amplitude are almost identical but for an overall rescaling, which illustrates how the modal evolution is not sensitive to the size of the initial disturbance in this parameter regime.

cross-over from the initial exponential decay of the quasi-mode to the protracted algebraic behaviour associated with the branch cut. Again, there are some differences with the analytical solution due to the finite domain of the numerical computation, but the branch-cut contribution $A_{\mathrm{bc}}$ is also only evaluated asymptotically.

For the low amplitudes at which the computations shown in figure 5 are performed, nonlinear effects are largely negligible; the dynamics is chiefly that of linear continuum damping. However, nonlinearity does become important at larger amplitude. In fact, there appears to be a threshold in the initial amplitude $A_{0}$ (which is about $A_{0}=0.025$ at these parameter values) above which continuum damping does not continue beyond an initial transient; see figure 6 . The termination of the decay of a sufficiently strong perturbation occurs because of the creation of a sustained nonlinear meander (see panel $b$ ). As shown by figure 6 , the meandering state contains vortices on either side 


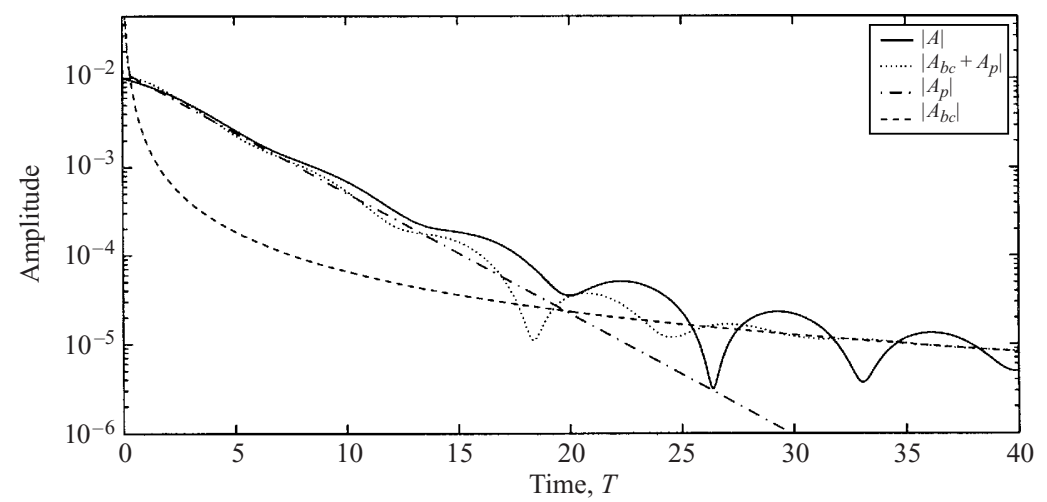

FiguRE 5. A stable jet with $\kappa=\gamma=1$ and $\lambda=0$ : the numerical, nonlinear solution, together with the analytical solution, $A_{p}(T)+A_{b c}(T)$. The contributions of the quasi-mode, $A_{p}(T)$, and the branch cut (at long times), $A_{b c}(T)$, are also shown individually.
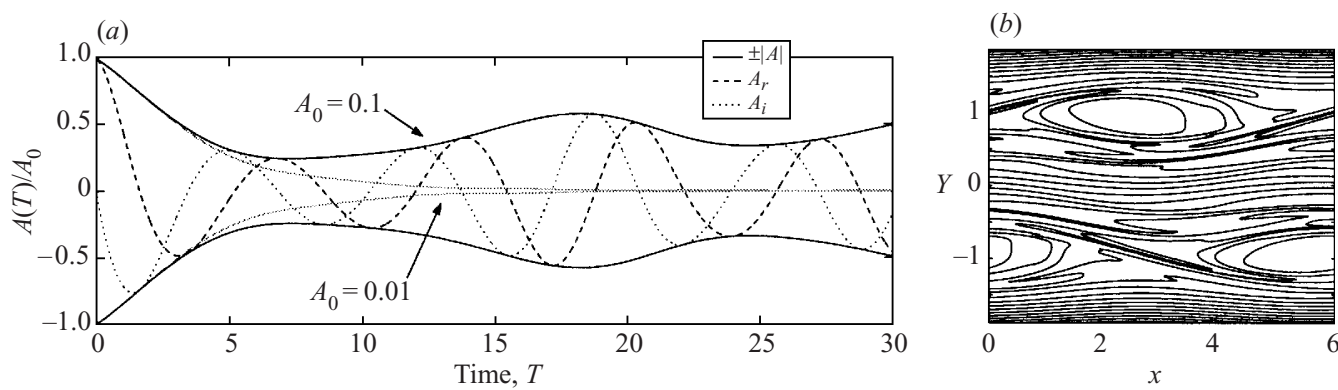

Figure 6. A stable jet with $\kappa=\gamma=1$. (a) Two computations of $A / A_{0}$ with $A_{0}=0.1$ and 0.01. $(b)$ The nonlinear state that emerges for $A_{0}=0.1$ : contours of constant, total vorticity $q(x, Y, T)=\zeta(x, Y, T)+\kappa\left(\varphi-Y^{3} / 3\right)+\gamma Y$ on the $(x, Y)$-plane at $T=30$. The equally spaced contour levels range from -0.9 to 0.9 in steps of 0.1 .

of the jet axis. These 'cat's eye' patterns are also generated by unstable meanders, as we see in the next subsection.

The termination of continuum damping by the formation of nonlinear meandering structure is analogous to the arrest of Landau damping in plasmas (Manfredi 1997) and of axisymmetrization in vortices (Balmforth et al. 2000). In those contexts nonlinear structures appear that again halt the return to the original equilibrium. For shear flows, such behaviour was also previously observed by Brunet \& Haynes (1995) on the axis of a balanced Poiseuille jet, and by Held \& Phillips (1987) for a flow over the surface of a sphere. This common property points to a universal behaviour for nonlinear continuum damping.

\subsection{Unstable jets}

In figure 7 we show an unstable jet with $\kappa=\gamma=-1$. This figure displays the growth of the mode amplitude and the resulting effect on the critical-layer vorticity distribution. As for the cases considered in paper I with separated critical layers, the mode grows until the amplitude saturates as a result of the twisting-up of the vorticity distribution into cat's eye patterns. Thereafter, the mode amplitude does not equilibrate at a steady level, but 'bounces' aperiodically. The novelty here is that two, staggered, chains of vortical cat's eyes form, straddling the jet axis. These are symptomatic of the merging critical layers for $(k, \beta) \rightarrow(\sqrt{6},-2)$. Figure 7 also illustrates the mixing action of 


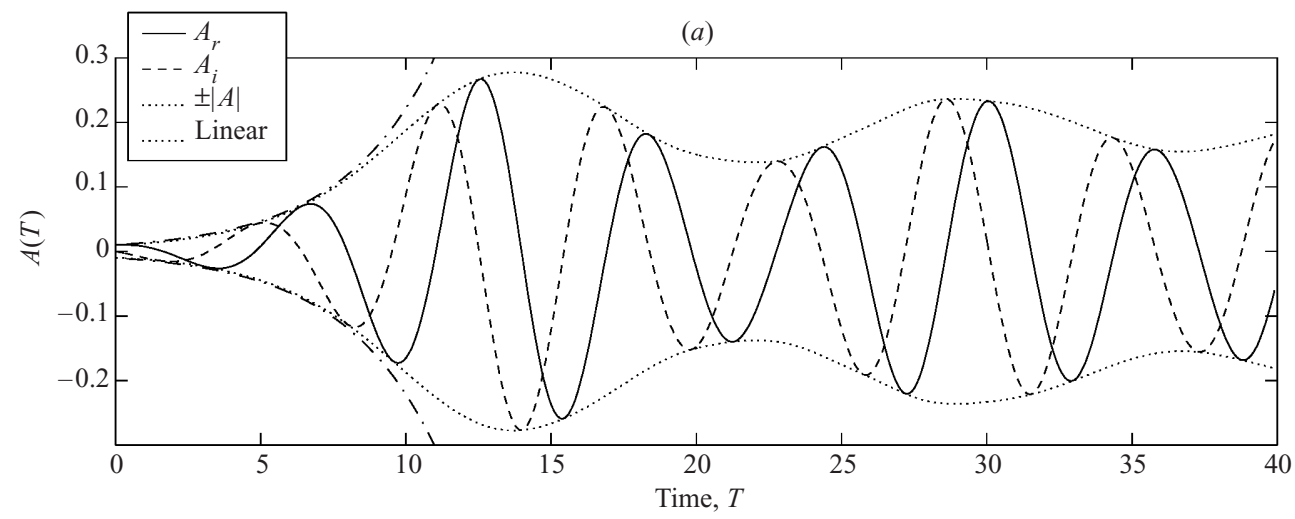

(b)
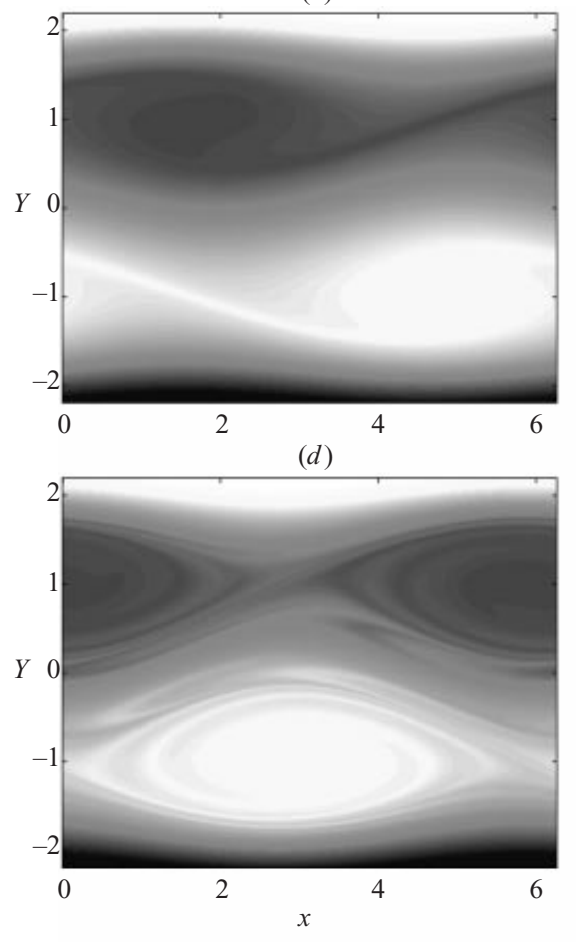

(c)

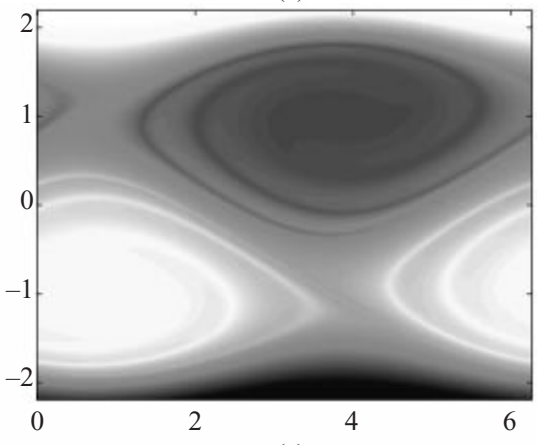

(e)

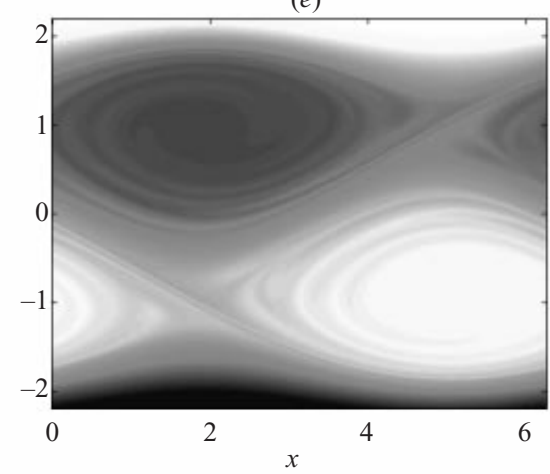

(f)
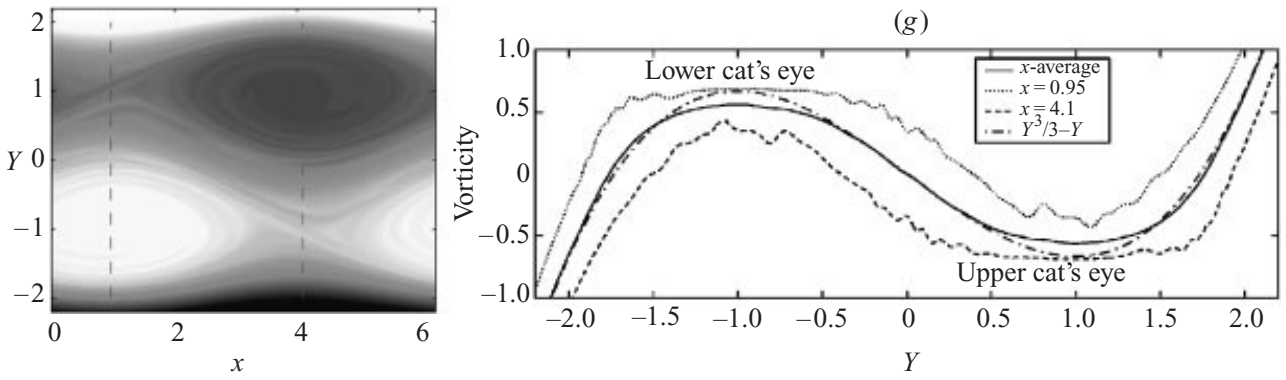

FIGURE 7. For caption see facing page. 

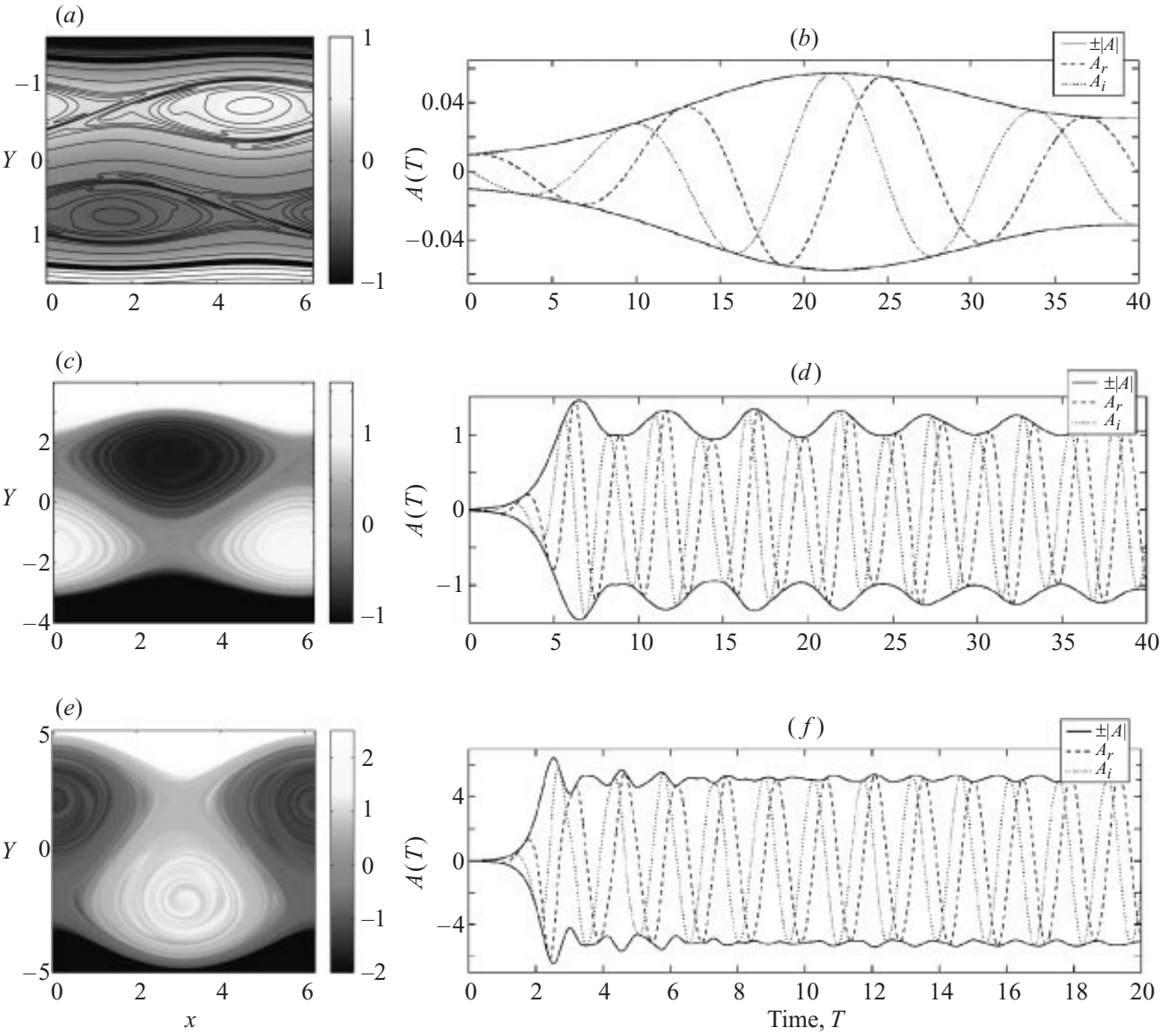

FiguRE 8. Unstable jets for $\gamma=-1, \lambda=10^{-5}$ and different values of $\kappa: \kappa=-2$ in $(a, b), \kappa=-1 / 2$ in $(c, d)$ and $\kappa=-1 / 4$ in $(e, f)$. $(a, c, e)$ Snapshots (at times $(a) T=40,(c) T=40,(e) T=32)$ of the total vorticity $q$ as densities on the $(x, Y)$-plane, with a shading scheme given by the keys. $(b, d, f)$ The history of the mode amplitude $A(T)$. In $(a)$, contours of constant vorticity are added to aid visualization.

the unstable mode, which homogenizes the vorticity within the cat's eyes and slightly flattens the $x$-averaged vorticity distribution. However, the mode saturates before that average becomes monotonic (an ingredient in mean-field or 'quasi-linear' theories).

An important parameter in the reduced model is $\kappa \propto-\beta_{2} / \mu^{2}$ (when we scale so that $\gamma=-1$ ). A decrease in $|\kappa|$ corresponds either to decreasing $\beta_{2}$, and moving horizontally to the left on the parameter plane, or to increasing $\mu$ and taking an downward path. Either way, we proceed towards the lower stability boundary. This leads to a change in the geometry of the nonlinear solutions that is illustrated in figure 8. At larger $|\kappa|$, there are separated cat's eye patterns bordering the meandering

Figure 7. An unstable jet with $\kappa=\gamma=-1$ and $\lambda=10^{-5}$. (a) The amplitude $A(T)$ and $(b-f)$ snapshots of the total vorticity $q=\zeta+\gamma Y+\kappa\left(\varphi-Y^{3} / 3\right)$ as densities on the $(x, Y)$-plane, with shading as given by the key and at times $(b) T=8,(c) 16,(d) 24,(e) 32,(f)$ 40. In $(a)$, the prediction of the linear theory is also shown. $(g)$ Cuts through the final vorticity distribution at $x=0.95$ and 4.1 (these are also marked in $f$ ), together with the $x$-averaged and initial distributions (the latter is given by $Y^{3} / 3-Y$ ). 


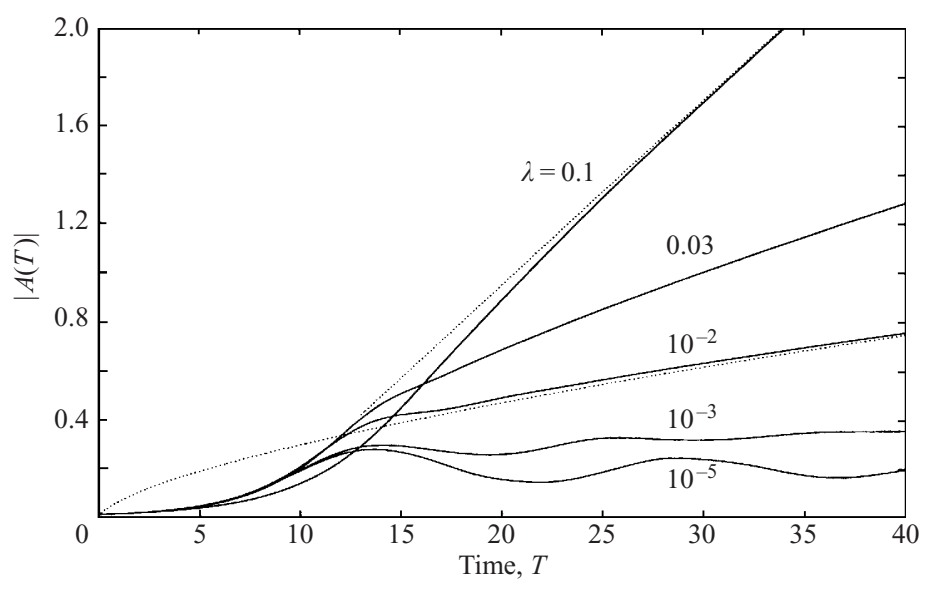

Figure 9. Plots of the mode amplitude $|A(T)|$ for $\kappa=\gamma=-1$ and several values of the viscosity parameter $\lambda$; the curves are labelled by the value of $\lambda$. Also shown is a curve with dependence $t^{2 / 3}$ fit to the case with $\lambda=10^{-2}$, and a linear fit to the case with $\lambda=0.1$.

core of the jet. But the cat's eyes approach the jet axis on lowering $|\kappa|$. Eventually, the vortex island chains displace the meandering core, and form a single row of vortices with the jet winding through them. This change in geometry implies a distinct change in the transport properties of the flow (del Castillo-Negrete \& Morrison 1993).

A second important parameter is the viscosity. In an isolated critical layer, viscosity plays a destructive role by permitting the vorticity to diffuse outwards (see paper I). Here, too, we find that viscosity allows the critical layer to spread diffusively, an effect that causes the mode amplitude to grow algebraically beyond the inviscid saturation level, see figure 9 (details of this process are given in the Appendix). For $\lambda=10^{-2}$, there is a clear $t^{2 / 3}$-dependence of $|A(T)|$, which mirrors the standard critical-layer problem (Churilov \& Shukhman 1996; paper I). However, the runs with higher viscosity diverge quicker. The $t^{2 / 3}$-dependence at $\lambda=10^{-2}$ arises because, for this case, the cat's eyes remain separated and spread individually for the length of the computation. The more viscous cases, however, diffuse faster and the cat's eyes merge at an early stage of the computation. The spread of the merged vorticity pattern then accelerates and $A(T)$ diverges approximately linearly with time (see the Appendix, which also derives some further properties of the quasi-steady patterns that form inside the expanding critical region).

\subsection{Numerical computations with the governing equations}

To compare with the asymptotic theory, we solve the governing equations (2.1)-(2.2) numerically as described in paper I. The scheme is pseudo-spectral and solves the equations on a doubly periodic domain; we tune the domain size so that an unstable mode with $k \approx \sqrt{6}$ has the minimum wavenumber in $x$, and initialize the computation with a disturbance taking the form of the unstable mode.

The numerical computations with (2.1)-(2.2) confirm the growth and saturation of a nonlinear meander. Moreover, the equilibrated vorticity distribution has the form of cat's eye patterns that approach one another and change geometry as we move left on the parameter plane, see figure 10 . Time series of the vorticity at mid-channel also show amplitude bounces much like the asymptotic theory (figure 11). Such time series were used in paper I in order to measure $A(T)$, and are a convenient measurement to take. Here, however, the time series is not equivalent to $A(T)$ because the measurement 

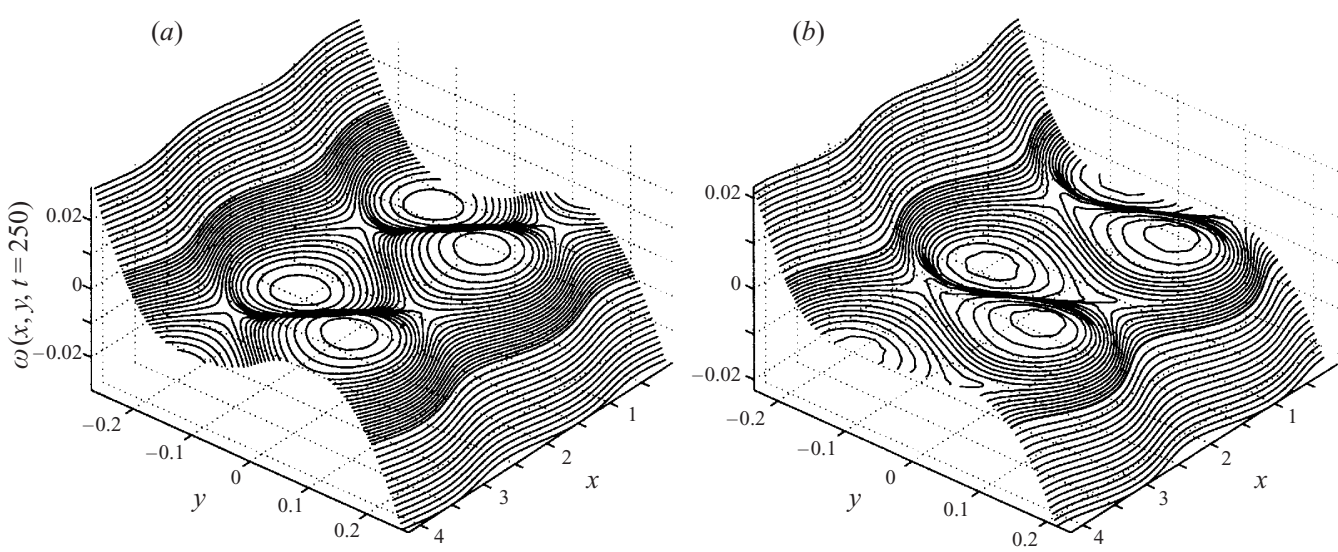

Figure 10. Numerical solutions of (2.1)-(2.2). Shown are contour plots of the total vorticity, $\omega+\beta y$ for two runs with $k=2.3$ and $(a) \beta=-1.85$ and $(b) \beta=-1.9 . t=250$ and $v=4.25 \times 10^{-6}$.

(a)

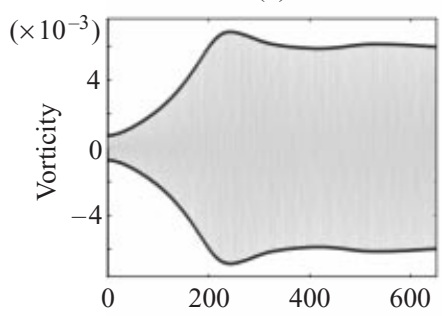

$(d)$

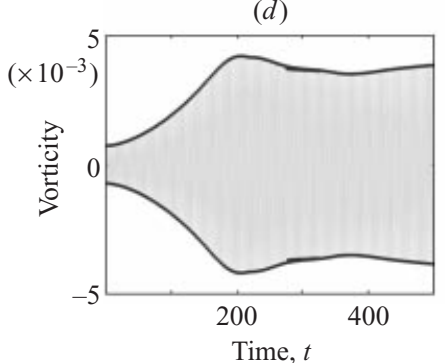

(b)

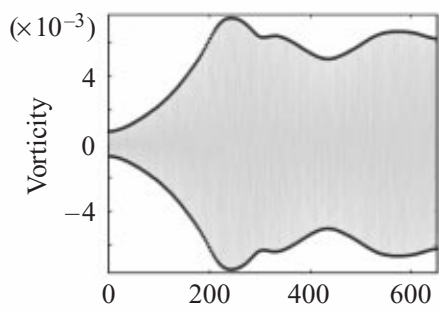

(e)

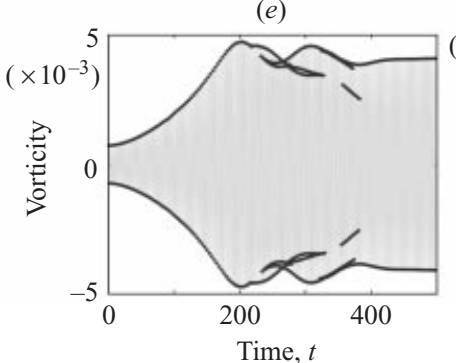

(c)

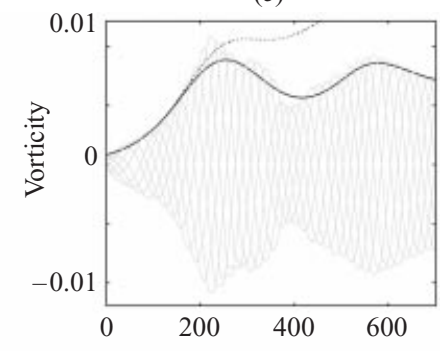

FIGURE 11. Time series of the vorticity at mid-channel: a numerical solution of (2.1)-(2.2) at $x=y=0$ for $k=2.3$ and $\beta=-1.85$, with $(a) v=4.25 \times 10^{-6}$ and $(b) v=1.7 \times 10^{-6}$. $(d, e)$ Similar plots for $\beta=-1.9$. The lighter curves show the actual time series of the vorticity, which contains the relatively fast oscillation of the unstable modes; the dots show the the maxima and minina of the oscillation. $(c, f)$ Analogous pictures constructed using the asymptotic theory (for which $\kappa=-1.34$ and $\kappa=-0.89$ respectively) with $\lambda=10^{-4}$. The lighter curves show time series from eight, equally spaced points at mid-channel $(Y=0)$; note that the asymptotic analysis filters the fast oscillation of the unstable mode. The heavier curves show the contribution of the mode (given by $\kappa \varphi$ ). Also shown by dotted lines are the mode contributions for computations with $\lambda=5.6 \times 10^{-3}$ in $(c)$, and $\lambda=1.1 \times 10^{-3}$ in $(f)$.

is now taken inside the critical region, and so the signal contains the signature of both the mode (as given by $\kappa \varphi$ ), and the twisting-up of the vorticity field (that is, $\zeta(x, 0, T))$. The latter introduces shorter time-scale variations into the signal, except for lower viscosities.

Given the time series of $\omega(0,0, t)$, we identify a 'first bounce amplitude' (the 

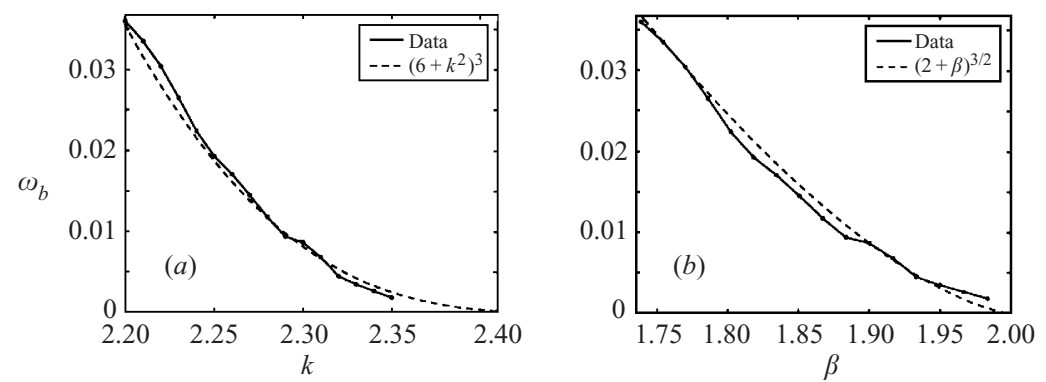

FIGURE 12. Scaling data from numerical computations with (2.1)-(2.2). The computations are performed along the curve $\beta=-1.9(k / 2.3)^{2}$ on the parameter plane, and the first bounce amplitude (the amplitude of the first maximum of $|\omega(0,0, t)|)$ is plotted against $(a) k$ and $(b) \beta$. The asymptotic scalings are also illustrated. $v=1.7 \times 10^{-6}$.

amplitude of the first maximum in $|\omega(0,0, t)|)$ that measures the saturation level. In figure 12, we plot this amplitude against $k$ and $\beta$ for a sequence of computations along a curve, $\beta=-1.9(k / 2.3)^{2}$, on the parameter plane. The 'scaling data' shown in the figure (that is, the dependence of the bounce amplitude on the distance to the point $(-2, \sqrt{6}))$ verifies the scalings of the asymptotic theory, namely $\omega \sim \epsilon^{3}$, or $\omega \sim\left(6-k^{2}\right)^{3}$ and $\omega \sim(2+\beta)^{3 / 2}$. Such scalings signify saturation levels that are even lower than the 'trapping scaling' of plasma physics and paper I (in which $\omega \sim \epsilon^{2}$ ), and significantly smaller than the 'Hopf scalings' of the strongly viscous problem (with $\omega \sim \epsilon^{1 / 2}$ ).

Despite several similarities, the numerical computations with (2.1)-(2.2) and asymptotic theory are not in complete agreement, partly because the computational runs are performed with fairly large values for $\epsilon\left(\epsilon \mu \approx 0.7\right.$ for $k=2.3$, and $\epsilon^{2} \beta_{2} \approx 0.1$ for $\beta=-1.9$ ). Notably, the asymptotic theory appears to overestimate the effect of viscosity: $\lambda=1.11 \times 10^{-3}$ and $5.3 \times 10^{-3}$ for the two cases of figure 11 with $v=1.7 \times 10^{-6}$. At such values of $\lambda$, the asymptotic theory predicts mode amplitudes that diverge relatively quickly (see panels $c$ and $f$ ), whereas the numerical computations show saturation near the first bounce amplitude even for larger viscosities. At the heart of this problem is that in the vicinity of the special point, the unstable mode is very slowly growing, and it is especially challenging to eliminate significant dissipation in the numerical computations with (2.1)-(2.2). Indeed, with a resolution of 512 gridpoints in $y$, it is difficult to reduce the viscosity sufficiently in order to enter the regime of the asymptotics whilst retaining a high enough viscosity to ensure the reliability of the computation (that is, that the computation is not dominated by a spectral filter; see paper I).

\section{Summary}

In this article we have derived a reduced model to describe the dynamics of a mode whose critical levels coalesce along the axis of a jet. We delineated the region of instability in this reduced model and explored both the linear and nonlinear dynamics that it captures. Numerical solutions of the governing equations illustrate and confirm much of the dynamics uncovered in the asymptotic analysis.

When the jet is stable, initial perturbations excite either neutral Rossby waves that ring persistently, or disturbances that can decay through the analogues of the KelvinOrr shear-tilting mechanism and Landau damping. In the latter case, the parabolic flow profile in the critical region has the novelty that the mean flow does not shear 
(a)

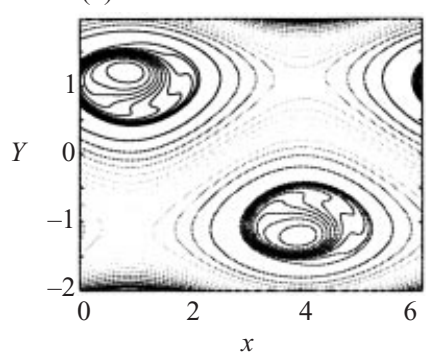

(b)

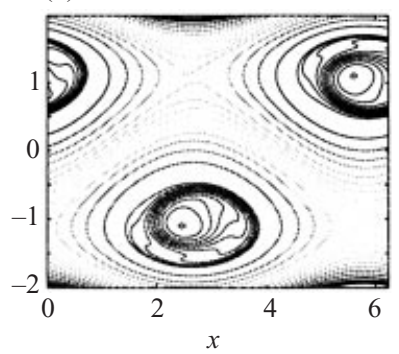

(c)

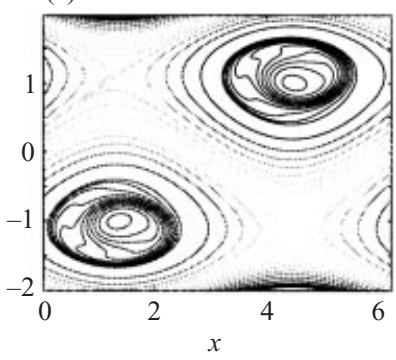

Figure 13. Satellite structures within the cat's eyes for $\kappa=-0.89$ and $\lambda=10^{-4}(\gamma=-1)$. Three snapshots of contours of constant total vorticity $q(x, Y, T)$ are displayed for times $(a) T=64$, (b) $68,(c) 72$.

apart disturbances as quickly as in linear shear flows, leading to a slower characteristic decay of low-amplitude perturbations (like $t^{-3 / 2}$ in the streamfunction; in shear flows without these parabolic points, streamfunction perturbations decay like $t^{-2}-$ Brown $\&$ Stewartson 1980). However, if the initial perturbations have sufficient amplitude, this decay is halted by the formation of cat's eye patterns in the vorticity field, as in plasmas (Manfredi 1997) and vortices (Balmforth et al. 2000). This behaviour has been found previously in some other shear flow studies, and suggests that 'nonlinear continuum damping' (phase mixing of nonlinear perturbations) occurs only below a threshold in initial amplitude for a wide variety of different physical systems. By contrast, no such threshold was found by Brunet \& Warn (1990), who studied the dynamics of a disturbance excited near the axis of a parabolic jet in which the vorticity gradient was exactly cancelled by a suitably chosen beta-parameter. Consequently, the fluid evolved at the shearless point without twisting up any background vorticity gradient, which is a key effect here. In Brunet \& Warn's special problem, perturbations decay even less quickly than in coalesced critical layers, with the result that nonlinear effects become important whatever the initial disturbance amplitude.

For unstable jets, the reduced model captures the merging of the critical levels of the unstable waves, their inviscid saturation, and the viscous spread of the critical layer. As in our earlier paper, inviscid saturation does not stabilize the mode amplitude at a steady level. Instead, there are aperiodic amplitude bounces (trapping oscillations) about the saturation level that continue for extended periods, if not indefinitely. These bounces are intimately connected with the appearance of secondary structures or satellites within the cat's eyes (see figure 13). Independently of any external perturbation, the bouncing breaks open the separating streamlines at the core of the jet that act as transport barriers, permitting a certain amount of fluid mixing (del Castillo-Negrete \& Morrison 1993).

Finally, the theory we have presented is strictly only valid for symmetrical jets because the scalings for the inner region fail if $U^{\prime \prime \prime}(0) \neq 0$. To explore the effect of the profile's skewness we can break the jet's symmetry perturbatively. The analysis then proceeds in a similar fashion, and furnishes a modified model,

$$
\begin{gathered}
\zeta_{T}+Y^{2} \zeta_{x}+\varphi_{x} \zeta_{Y}=\lambda \zeta_{Y Y}-\kappa \varphi_{T}-\gamma \varphi_{x}-\sigma Y \varphi_{x}, \\
\varphi=A \mathrm{e}^{\mathrm{i} x}+\text { c.c., } \quad A=\left\langle\mathrm{e}^{-\mathrm{i} x} \zeta\right\rangle,
\end{gathered}
$$

where $\sigma \propto U^{\prime \prime \prime}(0)$. The new term, $-\sigma Y \varphi_{x}$, does not change the linear stability theory but desymmetrizes the nonlinear vortical patterns that form. The system (7.1)-(7.2) 
provides a compact description of the dynamics of nearly inviscid jets near the threshold of instability, but we have not studied it in any detail (except in the symmetrical case) and leave its exploration for the future.

N. J. B. acknowledges an equipment grant from the Nuffield Foundation and a Faculty Research Grant from the University of California at Santa Cruz.

\section{Appendix. Quasi-steady critical layers}

In this Appendix we explore the system

$$
\begin{gathered}
\left(Y^{2}+\Theta_{T}\right) \zeta_{\xi}+2 a \zeta_{Y} \sin \xi-\lambda \zeta_{Y Y}=2 \kappa a_{T} \cos \xi-2 a\left(\gamma+\kappa \Theta_{T}\right) \sin \xi, \\
a=\langle\zeta \cos \xi\rangle, \quad 0=\langle\zeta \sin \xi\rangle,
\end{gathered}
$$

where $A=-a \mathrm{e}^{\mathrm{i} \Theta}$ and $\xi=x+\Theta$. This system arises when we transform into a frame moving with a (time-dependent) speed $-\Theta_{T}$ and drop the term $\zeta_{T}$, and can be obtained in a number of asymptotic limits of the reduced model.

We place the system into a more convenient form by defining

$$
\begin{gathered}
Y=\eta(2 a)^{1 / 3}, \quad \Omega=-\frac{\Theta_{T}}{(2 a)^{2 / 3}}, \quad \lambda=\frac{\Lambda}{(2 a)^{4 / 3}}, \\
\zeta=\frac{1}{2}(2 a)^{1 / 3}\left(\gamma+\kappa \Theta_{T}\right) \zeta_{2}-\frac{\kappa a_{T}}{(2 a)^{2 / 3}} \zeta_{1},
\end{gathered}
$$

where

$$
\left.\begin{array}{l}
\left(\eta^{2}-\Omega\right) \zeta_{1 \xi}+\zeta_{1 \eta} \sin \xi-\Lambda \zeta_{1 \eta \eta}=-2 \sin \xi \\
\left(\eta^{2}-\Omega\right) \zeta_{2 \xi}+\zeta_{2 \eta} \sin \xi-\Lambda \zeta_{2 \eta \eta}=-2 \cos \xi
\end{array}\right\}
$$

The two functions, $\zeta_{1}(\xi, \eta ; \Omega, \Lambda)$ and $\zeta_{2}(\xi, \eta ; \Omega, \Lambda)$ can, in principle, be tabulated along with the subsidiary quantities

$$
\Xi_{c j}(\Omega, \Lambda)=\int_{\infty}^{\infty} \mathrm{d} \eta \int_{0}^{2 \pi} \frac{\mathrm{d} \xi}{2 \pi} \zeta_{j} \cos \xi, \quad \Xi_{s j}(\Omega, \Lambda)=\int_{\infty}^{\infty} \mathrm{d} \eta \int_{0}^{2 \pi} \frac{\mathrm{d} \xi}{2 \pi} \zeta_{j} \sin \xi .
$$

Thence,

$$
a_{T}=\frac{\Xi_{s 1}(2 a)^{4 / 3}}{2 \kappa\left(\Xi_{s 2} \Xi_{c 1}-\Xi_{s 1} \Xi_{c 2}\right)}, \quad \gamma+\kappa \Theta_{T}=\frac{\Xi_{s 2}(2 a)^{1 / 3}}{\left(\Xi_{s 2} \Xi_{c 1}-\Xi_{s 1} \Xi_{c 2}\right)} .
$$

Because of the appearance of the additional parameter, $\Omega$, this system is more difficult to analyse than the corresponding one for standard critical layers (Churilov \& Shukhman 1996). We content ourselves here with a brief discussion of the limit $\Lambda \ll 1$, which can be studied further analytically.

In this limit, we define $\zeta_{1}=f(\xi, \eta)-2 \eta$ and $\zeta_{2}=g(\xi, \eta) / \Lambda$, in which case

$$
\Phi_{\eta} f_{\xi}-\Phi_{\xi} f_{\eta}=\Lambda f_{\eta \eta}, \quad \Phi_{\eta} g_{\xi}-\Phi_{\xi} g_{\eta}=\Lambda\left(g_{\eta \eta}-2 \cos \xi\right),
$$

where $\Phi=\eta^{3} / 3-\Omega \eta+2 \cos \xi$ is the total streamfunction. In both equations, we may regard the right-hand terms as perturbations of the left-hand side. Thence, to leading order in $\Lambda, f$ and $g$ are functions of $\Phi$ alone. By proceeding to the following order in $\Lambda$, and enforcing a solvability condition to ensure that the next-order solution remains periodic in $\xi$, we arrive at

$$
\frac{\mathrm{d}}{\mathrm{d} \Phi}\left[I(\Phi) f_{\Phi}\right]=0, \quad \frac{\mathrm{d}}{\mathrm{d} \Phi}\left[I(\Phi) g_{\Phi}-2 I_{1}(\Phi)\right]=0,
$$


(a)

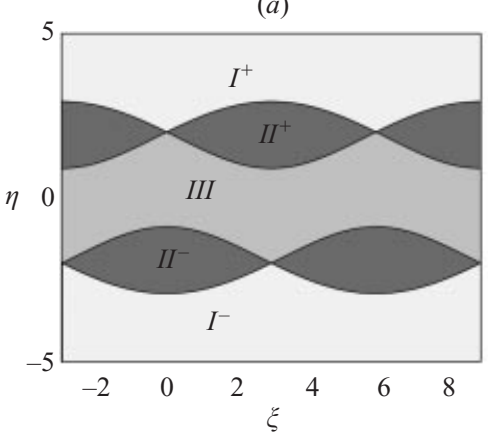

(b)

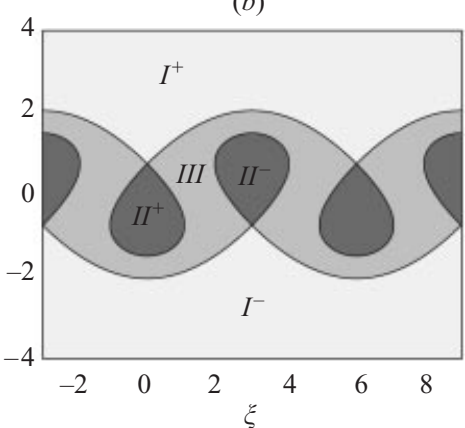

FIGURE 14. Illustration of the streamline pattern: (a) $\Omega=2$, and $(b) \Omega=3 / 4$.

(a)

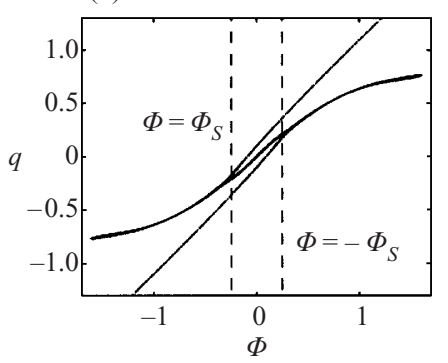

(b)

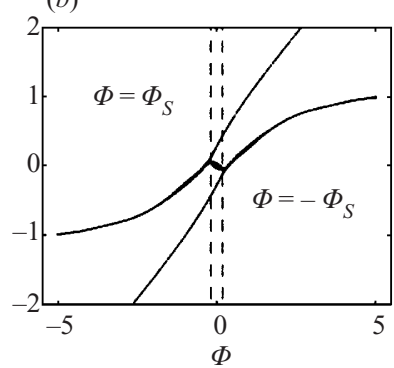

(c)

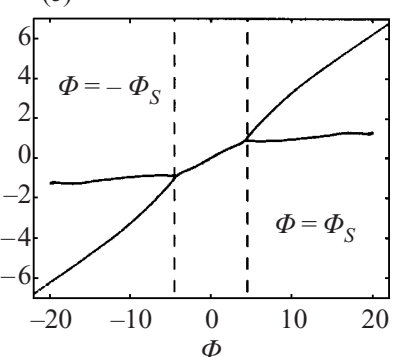

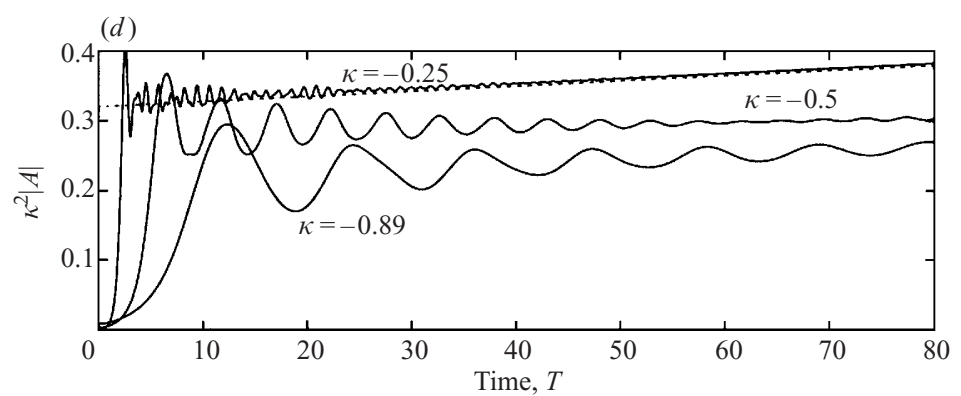

FIGURE 15. Plots of total streamfunction, $\Phi=Y^{3} / 3-\Omega Y-\varphi$, against total vorticity, $q$, at $T=80$ for $(a) \kappa=-0.89$, (b) $\kappa=-0.5$ and $(c) \kappa=-0.25\left(\gamma=-1\right.$ and $\left.\lambda=10^{-4}\right)$. The points are collected from various positions across the computational domain, and collapse very closely onto multi-branched curves. The vertical dashed lines show the separatrix values of $\Phi$. $(d)$ The history of the corresponding mode amplitudes, scaled by $\kappa^{2}$; the dotted line shows a linear fit to the case with $\kappa=-1 / 4$.

where

$$
I(\Phi)=\int_{C}\left[\eta(\xi, \Phi)^{2}-\Omega\right] \mathrm{d} \xi, \quad I_{1}(\Phi)=\int_{C} \eta(\xi, \Phi) \cos \xi \mathrm{d} \xi,
$$

and $C \equiv[0,2 \pi]$ if the streamline $\Phi=$ constant is open, and is around the streamline if it is closed.

At this stage it is helpful to consider in more detail the geometry of the streamline pattern. As illustrated in figure 14, depending on the value of $\Omega$, there are two possible configurations. In each configuration, the $(\xi, \eta)$-coordinate plane is divided into three characteristic regions: $I^{ \pm}$denote open streamline regions lying above and below the jet axis. $I I^{ \pm}$denote regions of closed streamlines corresponding to either the cat's 
eyes of separated critical layers, or to the tear-drop shaped areas in the alternatively connected jet configuration. Region $I I I$ is the central core of the meandering jet. In each of these regions, the functions $f(\Phi)$ and $g(\Phi)$ may take different forms; we require continuity across the separatrices (Brown \& Stewartson 1978), which we denote by $\Phi= \pm \Phi_{S} \equiv \pm 2\left(1-\Omega^{3 / 2} / 3\right)$. This produces a multi-valued relation between the streamfunction and total vorticity, which is also seen in numerical computations, see figure 15 .

Integration of (A9), and use of the symmetry of the solution and the boundary conditions, $f \rightarrow 2 \eta$ and $g$ finite as $\eta \rightarrow \pm \infty$, leads to

$$
\begin{gathered}
f= \begin{cases}f_{+}+4 \pi \int_{\Phi_{S}}^{\Phi} \mathrm{d} \Phi^{\prime} / I\left(\Phi^{\prime}\right) & \text { in } I^{+} \\
f_{+} & \text {in } I I^{+} \\
f_{+}+K_{1} \int_{\Phi_{S}}^{\Phi} \mathrm{d} \Phi^{\prime} / I\left(\Phi^{\prime}\right) & \text { in } I I I \\
-f_{+} & \text {in } I I^{-} \\
-f_{+}-4 \pi \int_{\Phi_{S}}^{-\Phi} \mathrm{d} \Phi^{\prime} / I\left(\Phi^{\prime}\right) & \text { in } I^{-},\end{cases} \\
g= \begin{cases}g_{+}+2 \int_{\Phi_{S}}^{\Phi} I_{1}\left(\Phi^{\prime}\right) \mathrm{d} \Phi^{\prime} / I\left(\Phi^{\prime}\right) & \text { in } I^{+} \\
g_{+}+2 \int_{\Phi_{S}}^{\Phi} I_{1}\left(\Phi^{\prime}\right) \mathrm{d} \Phi^{\prime} / I\left(\Phi^{\prime}\right) & \text { in } I I^{+} \\
g_{+}+2 \int_{\Phi_{S}}^{\Phi} I_{1}\left(\Phi^{\prime}\right) \mathrm{d} \Phi^{\prime} / I\left(\Phi^{\prime}\right)+K_{2} \int_{\Phi_{S}} \mathrm{~d} \Phi^{\prime} / I\left(\Phi^{\prime}\right) & \text { in } I I I \\
-g_{+}-2 \int_{\Phi_{S}}^{-\Phi} I_{1}\left(\Phi^{\prime}\right) \mathrm{d} \Phi^{\prime} / I\left(\Phi^{\prime}\right) & \text { in } I I^{-} \\
-g_{+}-2 \int_{\Phi_{S}}^{-\Phi} I_{1}\left(\Phi^{\prime}\right) \mathrm{d} \Phi^{\prime} / I\left(\Phi^{\prime}\right) & \text { in } I^{-},\end{cases}
\end{gathered}
$$

where

$$
2 f_{+}=K_{1} \int_{-\Phi_{S}}^{\Phi_{S}} \frac{\mathrm{d} \Phi}{I(\Phi)}, \quad 2 g_{+}=K_{2} \int_{-\Phi_{S}}^{\Phi_{S}} \frac{\mathrm{d} \Phi}{I(\Phi)}+\int_{-\Phi_{S}}^{\Phi_{S}} \frac{I_{1}(\Phi) \mathrm{d} \Phi}{I(\Phi)} .
$$

A property of the closed streamline region has also been used to eliminate an integration constant, which follows from a generalized form of the Prandtl-Batchelor theorem (Goldstein \& Hultgren 1988).

Neither of these solutions satisfies the requirement that $\zeta_{j} \rightarrow 0$ as $\eta \rightarrow \pm \infty$. In fact, both predict constant, finite vorticity in the far field, which calls for the introduction of an outer diffusion layer in which the vorticity is brought back to zero (Brown \& Stewartson 1978). A proper matching of the solution with the outer diffusion layer, together with a more detailed match across the separatrices, presumably determines the two constants, $f_{+}$and $g_{+}$. However, even without those details, we may still extract the asymptotic dependence of the functions $\Xi_{c j}(\Lambda, \Omega)$ and $\Xi_{s j}(\Lambda, \Omega), j=1$ and 2. First, because $\zeta_{1}=O(1)$ and $\zeta_{2}=O\left(\Lambda^{-1}\right)$, we expect that $\Xi_{c 1}=O(1)$ and $\Xi_{c 2}=O\left(\Lambda^{-1}\right)$. On the other hand, because $f$ and $g$ are functions of $\Phi$ to order $\Lambda$, it 
follows that $\Xi_{s 1}=O(\Lambda)$ and $\Xi_{s 2}=O(1)$. Therefore,

$$
a_{T}=\frac{1}{2 \kappa} \lambda\left(\frac{\Xi_{s 1} / \Lambda}{\Xi_{s 2} \Xi_{c 1}-\Xi_{s 1} \Xi_{c 2}}\right), \quad \gamma+\kappa \Theta_{T}=(2 a)^{1 / 3}\left(\frac{\Xi_{s 2}}{\Xi_{s 2} \Xi_{c 1}-\Xi_{s 1} \Xi_{c 2}}\right) .
$$

In each relation, the quantity in brackets is order one, though formally a function of $\Omega=-\kappa \Theta_{T} /(2 a)^{2 / 3}$. Two limits are accessible here. For $\Omega \gg 1$, regions $I^{ \pm}$and $I I I$ occupy most of the jet and the cat's eye regions become confined to small areas near $\eta= \pm \sqrt{\Omega}$. Each cat's eye is then decoupled from the other, and the problem reduces to the standard quasi-steady critical-layer problem (for which $a \sim t^{2 / 3}$; Goldstein \& Hultgren 1988; Churilov \& Shukhman 1996). On the other hand, if $\Omega \rightarrow 0$ (which arises when $a$ becomes large), the integrals limit to constants, and we observe that $a_{T} \approx$ constant. Hence the mode amplitude grows linearly with time, as displayed by the time series for $\kappa=-1 / 4$ shown in figure 15 .

\section{REFERENCES}

Abramowitz, M. \& Stegun, I. 1972 Handbook of Mathematical Function. Wiley Interscience.

Balmforth, N. J. 1998 Stability of vorticity defects in viscous shear. J. Fluid Mech. 357, 199-224.

Balmforth, N. J., Llewellyn Smith, S. G. \& Young, W. R. 2000 Disturbing vortices. J. Fluid Mech. 426, 95-133.

Balmforth, N. J. \& Piccolo, C. 2001 The onset of meandering in a barotropic jet. J. Fluid Mech. 449, 85-114 (paper I).

Brown, S. N. \& Stewartson, K. 1978 The evolution of the critical layer of a Rossby wave. Part II. Geophys. Astrophys. Fluid Dyn. 10, 1-24.

Brown, S. N. \& Stewartson, K. 1980 On the algebraic decay of disturbances in the stratified shear flow. J. Fluid Mech. 100, 811-816.

Brunet, G. \& Haynes, P. H. 1995 The nonlinear evolution of disturbances to a parabolic jet. J. Atmos. Sci. 52, 464-477.

Brunet, G. \& Warn, T. 1990 Rossby wave critical layers on a jet. J. Atmos. Sci. 47, 1173-1178.

del Castillo-Negrete, D. \& Morrison, P. J. 1993 Chaotic advection by Rossby waves in shear flow. Phys. Fluids A 5 948-965.

Churilov, S. M. \& Shukhman, I. G. 1996 The nonlinear critical layer resulting from the spatial or temporal evolution of weakly unstable disturbances in shear flow. J. Fluid Mech. 318, 189-221.

Drazin, P. G., Beaumont, D. N. \& CoAKer, S. A. 1982 On Rossby waves modified by basic shear, and barotropic instability. J. Fluid Mech. 124, 439-456.

Goldstein, M. E. \& Hultgren, L. S. 1988 Nonlinear spatial evolution of an externally excited instability wave in a free shear layer. J. Fluid Mech. 197, 259-330.

Held, I. M. \& Phillips, P. J. 1987 Linear and nonlinear barotropic decay on the sphere. J. Atmos. Sci. 44, 200-207.

Howard, L. N. \& Drazin, P. G. 1964 On instability of parallel flow of inviscid fluid in a rotating system with variable coriolis parameter. J. Math. Phys. 43, 83-99.

Mallier, R. \& Davis, A. M. J. 1999 Nonlinear evolution of singular disturbances to the Bickley jet. Stud. Appl. Maths 103, 149-181.

MANFredi, G. 1997 Long-time behaviour of nonlinear Landau damping. Phys. Rev. Lett. 79, 28152819.

Maslowe, S. A. 1991 Barotropic instability of the bickley jet. J. Fluid Mech. 229, 417-426.

Solomon, T. H., Holloway, W. J. \& Swinney, H. 1993 Shear flow instabilities and Rossby waves in barotropic fluid in a rotating annulus. Phys. Fluids A 5, 1971-1988.

Swaters, G. R. 1999 On the evolution of near-singular modes of the Bickley jet. Phys. Fluids 11, 2546-2555. 